\title{
Allenylphosphonates with a 1,3,2-dioxaphosphorinane ring: Synthesis, structures, stability and utility
}

\author{
N N BHUVAN KUMAR, MANAB CHAKRAVARTY, N SATISH KUMAR, \\ K V SAJNA and K C KUMARA SWAMY* \\ School of Chemistry, University of Hyderabad, Hyderabad 500046 \\ e-mail: kckssc@uohyd.ernet.in; kckssc@yahoo.com
}

MS received 17 July 2008; revised 15 October 2008

\begin{abstract}
Synthesis, structures and stability (thermal and air) of allenylphosphonates of the type $\left(\mathrm{OCH}_{2} \mathrm{CMe}_{2} \mathrm{CH}_{2} \mathrm{O}\right) \mathrm{P}(\mathrm{O}) \mathrm{C}(\mathrm{R})=\mathrm{C}=\mathrm{CR}^{\prime} \mathrm{R}^{\prime \prime}$ and $\left[\mathrm{R}^{\prime \prime} \mathrm{R}^{\prime} \mathrm{C}=\mathrm{C}=\mathrm{C}(\mathrm{R}) \mathrm{P}(\mathrm{O})\left(\mathrm{OCH}_{2}\right)_{2}\right]_{2} \mathrm{C}$ are discussed. Thermally activated dimerization (cycloaddition) of $\left(\mathrm{OCH}_{2} \mathrm{CMe}_{2} \mathrm{CH}_{2} \mathrm{O}\right) \mathrm{P}(\mathrm{O}) \mathrm{C}(\mathrm{H})=\mathrm{C}=\mathrm{CMe}_{2}$ leads to the phosphonocyclobutane $\left[\left(\mathrm{OCH}_{2} \mathrm{CMe}_{2} \mathrm{CH}_{2} \mathrm{O}\right) \mathrm{P}(\mathrm{O}) \mathrm{C}(\mathrm{H})=\right]_{2}\left[\mathrm{C}-\mathrm{CMe}_{2}\right]_{2}(\mathbf{1 8})$. Many of these allenes undergo addition of diethylamine to lead to enaminophosphonates that are readily hydrolysed by water to lead to $\beta$ ketophosphonates. The latter compounds are useful as Horner-Wadsworth-Emmons (HWE) reagents. Molecular structures of $\left(\mathrm{OCH}_{2} \mathrm{CMe}_{2} \mathrm{CH}_{2} \mathrm{O}\right) \mathrm{P}(\mathrm{O}) \mathrm{C}\left(\mathrm{CH}_{2} \mathrm{OH}\right)=\mathrm{C}=\mathrm{CH}_{2}(\boldsymbol{6}),\left[\mathrm{H}_{2} \mathrm{C}=\mathrm{C}=\mathrm{C}(\mathrm{H}) \mathrm{P}(\mathrm{O})\left(\mathrm{OCH}_{2}\right)_{2}\right]_{2} \mathrm{C}(\mathbf{9})$, $\left(\mathrm{OCH}_{2} \mathrm{CMe}_{2} \mathrm{CH}_{2} \mathrm{O}\right) \mathrm{P}(\mathrm{O}) \mathrm{CH}=\mathrm{C}=\mathrm{CH}_{2}(\mathbf{1 2}),\left[\left(\mathrm{OCH}_{2} \mathrm{CMe}_{2} \mathrm{CH}_{2} \mathrm{O}\right) \mathrm{P}(\mathrm{O}) \mathrm{C}(\mathrm{H})=\right]_{2}\left[\mathrm{C}-\mathrm{CMe}_{2}\right]_{2}$ (18), and the $\beta$-ketophosphonate $\left.\left(\mathrm{OCH}_{2} \mathrm{CMe}_{2} \mathrm{CH}_{2} \mathrm{O}\right) \mathrm{P}(\mathrm{O}) \mathrm{CH}_{2}\right)-\mathrm{C}(\mathrm{O}) \mathrm{CHMe}_{2}(\mathbf{2 4})$ have been determined. In compound 6 , intermolecular hydrogen bonding between the phosphoryl oxygen and the hydroxyl group leading to an infinite chain is observed. In $\mathbf{6}$, there is a significant deviation $\left(\mathrm{ca} 7^{\circ}\right)$ from the orthogonality expected between the planes containing 4 atoms of (a) $\mathrm{H}_{2} \mathrm{C}=\mathrm{C}$ and (b) $\mathrm{C}=\mathrm{C}(\mathrm{H}) \mathrm{P}$ in the allene part. In 9, weak $\mathrm{C}-\mathrm{H} \ldots \mathrm{O}$ interaction between the phosphoryl oxygen atom and a $\mathrm{CH}_{2}$ proton of the six-membered ring is present.
\end{abstract}

Keywords. Allenylphosphonate; phosphonyl-cyclobutane; cycloaddition; X-ray crystal structure; Horner-Wadsworth-Emmons reaction.

\section{Introduction}

Allenes have two cumulative but orthogonal $\mathrm{C}=\mathrm{C}$ bonds that can undergo a variety of reactions and hence can be transformed into a variety of other useful organic compounds. ${ }^{1}$ Phosphorylated allenes (allenylphosphonates) that constitute a subclass of allenes are also synthetically useful. ${ }^{2}$ In our work on organophosphonates, ${ }^{3}$ we needed to synthesize different allenylphosphonates as substrates for a variety of reactions. ${ }^{4-7}$ Three synthetically useful reactions using allenylphosphonates developed from our laboratory are shown in scheme $1 .{ }^{4 a, 6,7}$ However, the number of readily accessible allenylphosphonates that are convenient to handle as solids is rather limited. Solid state structural studies on such phosphonates are also rather limited ${ }^{8}$ In addition, very little information is available on the thermal stability as well as allenic group reactivity (in

\footnotetext{
*For correspondence
}

allenylphosphonates) towards water. These are some of the points we address in this paper.

\section{Experimental}

Chemicals were procured from Aldrich or from local manufacturers; they were purified when required according to standard procedures. ${ }^{9}$ All reactions, unless stated otherwise, were performed in a dry nitrogen atmosphere. ${ }^{1} \mathrm{H},{ }^{13} \mathrm{C}$ and ${ }^{31} \mathrm{P}\{\mathrm{H}\}$ NMR spectra were recorded using a 200 or a $400 \mathrm{MHz}$ spectrometer in $\mathrm{CDCl}_{3}$ (unless stated otherwise) with shifts referenced to $\mathrm{SiMe}_{4}(\delta=0)$ or $85 \% \mathrm{H}_{3} \mathrm{PO}_{4}(\delta=0)$. Infrared spectra were recorded on a JASCO FT/IR 5300 FTIR spectrometer. Mass spectra were recorded using a LCMS 2010A. LC-MS data were obtained using electrospray ionization (positive mode) on a $\mathrm{C}-18$ column at a flow rate $0.2 \mathrm{~mL} / \mathrm{min}$ using $\mathrm{MeOH} /$ water $(90: 10)$ as eluent. Melting points were determined by using a local hot-stage melting point apparatus and are uncorrected. Microanalyses were performed using a Thermo Finnigan EA1112 analyser. Com- 
(i)

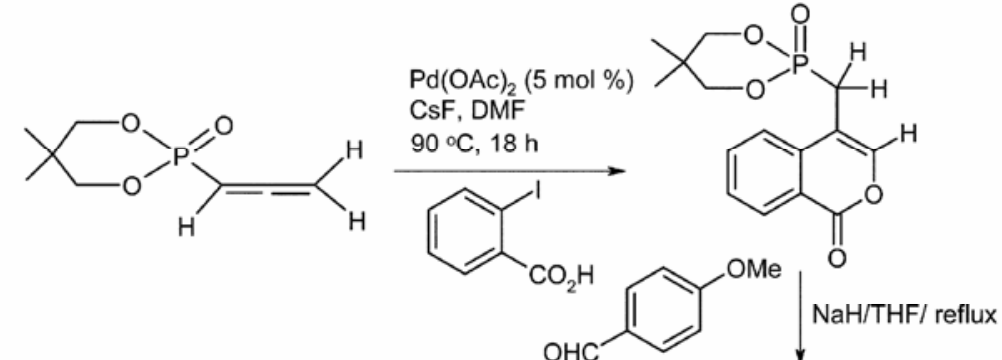

(ii)

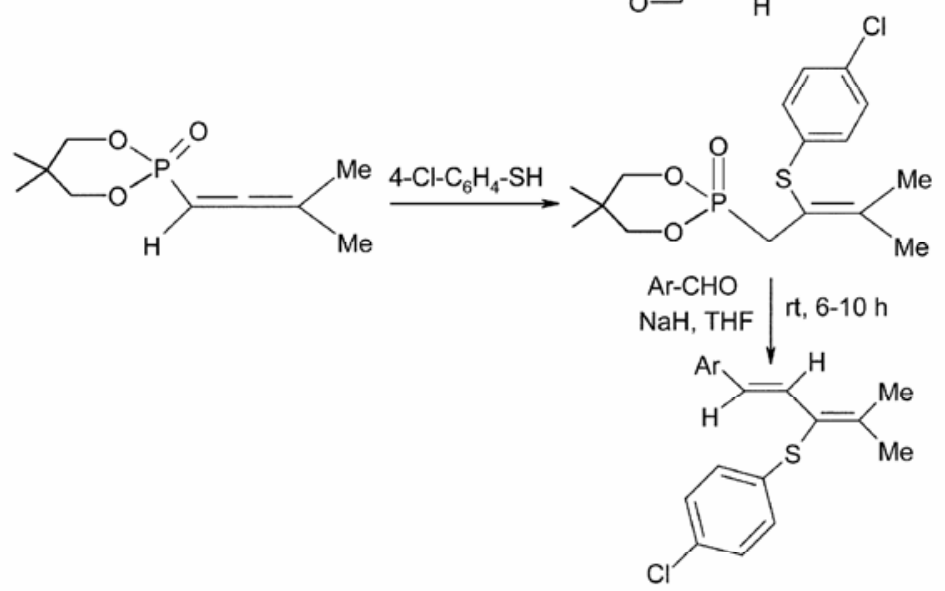

(iii)

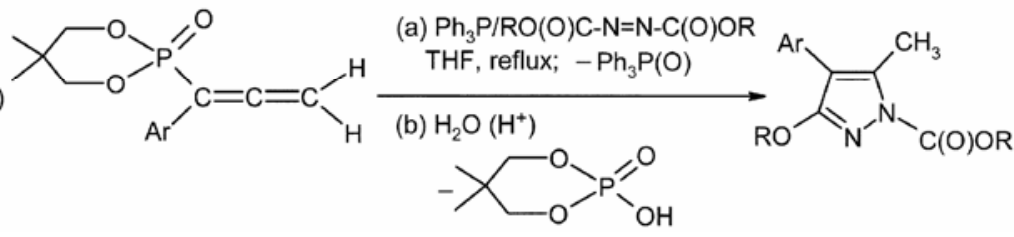

Scheme 1.

pounds $\mathbf{1}^{10}$ and $\mathbf{2}^{11}$ were prepared by methods reported in the literature.

\subsection{Synthesis of allenylphosphonates}

$\left(\mathrm{OCH}_{2} \mathrm{CMe}_{2} \mathrm{CH}_{2} \mathrm{O}\right) \mathrm{P}\left(\mathrm{CR}^{3}=\mathrm{C}=\mathrm{CR}^{1} \mathrm{R}^{2}\right)(3-5)$

To a solution of substituted propargyl alcohol $\mathrm{R}^{3} \mathrm{C} \equiv \mathrm{CCR}^{1} \mathrm{R}^{2} \mathrm{OH}(23.5 \mathrm{mmol})$ in dry THF $(50 \mathrm{~mL})$ was added triethylamine $(2.47 \mathrm{~g}, 3.40 \mathrm{~mL}, 23.5 \mathrm{mmol})$, the mixture stirred for $5 \mathrm{~min}$, and then $\left(\mathrm{OCH}_{2} \mathrm{CMe}_{2}\right.$ $\left.\mathrm{CH}_{2} \mathrm{O}\right) \mathrm{PCl}(1)(4.11 \mathrm{~g}, 3.37 \mathrm{~mL}, 23.5 \mathrm{mmol})$ in $\mathrm{THF}$ $(20 \mathrm{~mL})$ was added drop-wise $(\sim 0.5 \mathrm{~h})$ at $0^{\circ} \mathrm{C}$. The contents were brought to room temperature, stirred further for $1 \mathrm{~h}$, and then refluxed for $16 \mathrm{~h}$. Triethylamine hydrochloride formed was filtered off and solvent removed in vacuo from the filtrate. Allenes 3-5 were purified by column chromatography (silica gel; ethyl acetate-hexane $2: 3$ ).
2. la $\left(\mathrm{OCH}_{2} \mathrm{CMe}_{2} \mathrm{CH}_{2} \mathrm{O}\right) \mathrm{PC}\left[\left(\mathrm{CH}_{2}\right)_{4} \mathrm{CH}_{3}\right]=\mathrm{C}=\mathrm{CH}_{2}$ (3): Yield: $4.87 \mathrm{~g}(80 \%$; using $23.5 \mathrm{mmol}$ of 1). M.p.: 57-60 ${ }^{\circ} \mathrm{C}$. IR (KBr): 3071, 2928, 2855, 1944, 1769, 1470, 1372, 1258, 1047, 993, $824 \mathrm{~cm}^{-1}$. ${ }^{1} \mathrm{H}$ NMR: $\delta$ $0.90\left(t,{ }^{3} J(\mathrm{H}-\mathrm{H})=6.8 \mathrm{~Hz}, 3 \mathrm{H}, \mathrm{CH}_{2} \mathrm{CH}_{3}\right), 0.99$ and $1.20 \quad\left(2 s, 6 \mathrm{H}, \quad \mathrm{C}\left(\mathrm{CH}_{3}\right)_{2}\right), 1.33-1.35 \quad(m, 4 \mathrm{H}$, $\left.\mathrm{CH}_{2} \mathrm{CH}_{2} \mathrm{CH}_{3}\right), 1.52-1.56\left(m, 2 \mathrm{H}, \mathrm{PCCCH}_{2}\right), 2 \cdot 16-$ $2.23\left(m, 2 \mathrm{H}, \mathrm{PCCH}_{2}\right), 3.93-4.09\left(m, 4 \mathrm{H}, \mathrm{OCH}_{2}\right)$, $5.04\left(t d,{ }^{4} J(\mathrm{P}-\mathrm{H})=13.6 \mathrm{~Hz},{ }^{5} J(\mathrm{H}-\mathrm{H}) \sim 3.1 \mathrm{~Hz}, 2 \mathrm{H}\right.$, $\left.=\mathrm{CH}_{2}\right) \cdot{ }^{13} \mathrm{C} \mathrm{NMR}: \delta$ 14.0 $\left(\mathrm{s}, \mathrm{CH}_{2} \mathrm{CH}_{3}\right), 21.0$ and 21.7 $\left(2 s, \mathrm{C}\left(\mathrm{CH}_{3}\right)_{2}\right), 22 \cdot 3 \quad\left(s, \mathrm{CH}_{2} \mathrm{CH}_{3}\right), 27 \cdot 6\left(d,{ }^{4} J(\mathrm{P}-\right.$ $\left.\mathrm{C})=6 \cdot 1 \mathrm{~Hz}, \mathrm{CH}_{2} \mathrm{CH}_{2} \mathrm{CH}_{3}\right), 27 \cdot 8\left(d,{ }^{3} \mathrm{~J}(\mathrm{P}-\mathrm{C})=6 \cdot 1 \mathrm{~Hz}\right.$, $\left.\mathrm{PCCH}_{2} \mathrm{CH}_{2}\right), 31.2\left[s\left({ }^{2} \mathrm{~J}(\mathrm{P}-\mathrm{C})<2.0 \mathrm{~Hz}\right), \mathrm{PCCH}_{2}\right]$, $32.6\left(d,{ }^{3} J(\mathrm{P}-\mathrm{C})=7.3 \mathrm{~Hz}, C\left(\mathrm{CH}_{3}\right)_{2}\right), 76.7\left(s, 2 \mathrm{OCH}_{2}\right)$, $77.3\left(s,=C \mathrm{H}_{2}\right), 92.0\left(d,{ }^{1} J(\mathrm{P}-\mathrm{C})=180.7 \mathrm{~Hz}, \mathrm{PC}\right)$, $211.5\left(d,{ }^{2} J(\mathrm{P}-\mathrm{C})=6.1 \mathrm{~Hz}, \mathrm{PC} C\right)$ [the assignment of the alkyl carbons is tentative]. ${ }^{31} \mathrm{P} \mathrm{NMR}: \delta 11 \cdot 3$. $\mathrm{LC} / \mathrm{MS} m / z 259[\mathrm{M}+1]^{+}$. 
$2.1 \mathrm{~b} \quad\left(\mathrm{OCH}_{2} \mathrm{CMe}_{2} \mathrm{CH}_{2} \mathrm{O}\right) \mathrm{PC}\left[\left(\mathrm{CH}_{2}\right)_{5} \mathrm{CH}_{3}\right]=\mathrm{C}=\mathrm{CH}_{2}$ (4): Yield: $4.99 \mathrm{~g}(78 \%$ using $23.5 \mathrm{mmol}$ of 1). M.p.: 58-61 ${ }^{\circ} \mathrm{C}$; IR (KBr): $3075,2926,2857,1943,1773$, 1470, 1372, 1258, 1047, 997, $826 \mathrm{~cm}^{-1}$. ${ }^{1} \mathrm{H}$ NMR: $\delta$ $0.89\left(t,{ }^{3} J(\mathrm{H}-\mathrm{H}) \sim 6.8 \mathrm{~Hz}, 3 \mathrm{H}, \mathrm{CH}_{2} \mathrm{CH}_{3}\right), 0.99$ and $1.19\left(2 s, 6 \mathrm{H}, \mathrm{C}\left(\mathrm{CH}_{3}\right)_{2}\right), 1.30-1.37\left(m, 6 \mathrm{H}, \mathrm{CH}_{2} \mathrm{CH}_{2}\right.$ $\left.\mathrm{CH}_{2} \mathrm{CH}_{3}\right), 1.50-1.55\left(\mathrm{~m}, 2 \mathrm{H}, \mathrm{PCCCH} \mathrm{H}_{2}\right), 2 \cdot 16-2.21$ $\left(m, 2 \mathrm{H}, \mathrm{PCCH}_{2}\right), 3.92-4.09\left(m, 4 \mathrm{H}, \mathrm{OCH}_{2}\right), 5.04(d$, $\left.{ }^{4} \mathrm{~J}(\mathrm{P}-\mathrm{H})=13.6 \mathrm{~Hz}, 2 \mathrm{H},=\mathrm{CH}_{2}\right) \cdot{ }^{13} \mathrm{C} \mathrm{NMR}: \delta 14.0(s$, $\left.\mathrm{CH}_{2} \mathrm{CH}_{3}\right), 21.0$ and $21.7\left(2 s, \mathrm{C}\left(\mathrm{CH}_{3}\right)_{2}\right), 22.5(s$, $\left.\mathrm{CH}_{2} \mathrm{CH}_{3}\right), 27.77,27.83,28.7$ and $31.5\left(4 s, \mathrm{PCCH}_{2}\right.$ $\left.\mathrm{CH}_{2} \mathrm{CH}_{2} \mathrm{CH}_{2} \mathrm{CH}_{2} \mathrm{CH}_{3}\right), 32.5 \quad\left(d,{ }^{3} J(\mathrm{P}-\mathrm{C})=6.6 \mathrm{~Hz}\right.$, $\left.C\left(\mathrm{CH}_{3}\right)_{2}\right), 76.6$ and $76.7\left(2 s, \mathrm{OCH}_{2}\right), 77.2\left(d,{ }^{3} J(\mathrm{P}-\right.$ $\left.\mathrm{C})=15.8 \mathrm{~Hz},=\mathrm{CH}_{2}\right), 92.0\left(d,{ }^{1} J(\mathrm{P}-\mathrm{C})=181.3 \mathrm{~Hz}\right.$, PC), $211.5\left(d,{ }^{2} J(\mathrm{P}-\mathrm{C})=5.9 \mathrm{~Hz}, \mathrm{PC} C\right) .{ }^{31} \mathrm{P}$ NMR: $\delta$ 12.2. Anal. Calc. for $\mathrm{C}_{14} \mathrm{H}_{25} \mathrm{O}_{3} \mathrm{P}: \mathrm{C}, 61.75 ; \mathrm{H}, 9.25$. Found: C, 61.76; H, 9.23.

2.1c $\left(\mathrm{OCH}_{2} \mathrm{CMe}_{2} \mathrm{CH}_{2} \mathrm{O}\right) \mathrm{PC}(\mathrm{Ph})=\mathrm{C}=\mathrm{CH}(\mathrm{CH}=\mathrm{CHMe})$ (5): Yield: $5.01 \mathrm{~g}$ (70\% using $23.5 \mathrm{mmol}$ of 1). M.p.: $122-124^{\circ} \mathrm{C}$; IR (KBr): 2961, 2926, 1927, 1815, 1597, 1491, 1476, 1456, 1267, 1055, $1003 \mathrm{~cm}^{-1} .{ }^{1} \mathrm{H}$ NMR: $\delta 0.91$ and $1.33\left(2 s, 6 \mathrm{H}, \mathrm{C}\left(\mathrm{CH}_{3}\right)_{2}\right), 1.85(d d$, $\left.{ }^{3} J(\mathrm{H}-\mathrm{H})=5.3 \mathrm{~Hz}, \quad{ }^{4} J(\mathrm{H}-\mathrm{H})=3.5 \mathrm{~Hz}, \quad 3 \mathrm{H}, \quad \mathrm{CH}_{3}\right)$, 3.96-4.02 (m, 4H, $\left.\mathrm{OCH}_{2}\right), 5.92-5.97 \quad(m, 2 \mathrm{H}$, $\left.\mathrm{CH}=\mathrm{CH}-\mathrm{CH}_{3}\right), \quad 6.44 \quad\left(d d, 1 \mathrm{H},{ }^{4} J(\mathrm{P}-\mathrm{H}) \sim 12.0 \mathrm{~Hz}\right.$, $\left.{ }^{3} J(\mathrm{H}-\mathrm{H}) \sim 6.0 \mathrm{~Hz}, \quad \mathrm{C}=\mathrm{C}=\mathrm{C} H\right), 7.28-7.63 \quad(m, 5 \mathrm{H}$, $\mathrm{ArC}) .{ }^{13} \mathrm{C}$ NMR $\left(100 \mathrm{MHz}, \mathrm{CDCl}_{3}\right): \delta 18.5(s$, $\left.=\mathrm{C}(\mathrm{H}) \mathrm{CH}_{3}\right), 20.9$ and $22.0\left(2 s, \mathrm{C}\left(\mathrm{CH}_{3}\right)_{2}\right), 32.6(d$, $\left.{ }^{3} J(\mathrm{P}-\mathrm{C})=7.0 \mathrm{~Hz}, \quad C\left(\mathrm{CH}_{3}\right)_{2}\right), 77.2$ and $77.6 \quad(2 d$, $\left.{ }^{2} J(\mathrm{P}-\mathrm{C})=7.0 \mathrm{~Hz}, \mathrm{OC} \mathrm{H}_{2}\right), 97.7\left(d,{ }^{3} J(\mathrm{P}-\mathrm{C})=16.0 \mathrm{~Hz}\right.$, $\mathrm{C}=\mathrm{C}=C \mathrm{H}), 97.3\left(d,{ }^{1} J(\mathrm{P}-\mathrm{C})=181.0 \mathrm{~Hz}, \mathrm{PC}\right), 122.8$ $\left(d,{ }^{4} J(\mathrm{P}-\mathrm{C})=10 \cdot 0 \mathrm{~Hz}, \mathrm{C}=\mathrm{C}=\mathrm{CH}-C \mathrm{H}\right), 127 \cdot 8,127 \cdot 9$, $128 \cdot 1,128 \cdot 8,131 \cdot 2_{6}, 131 \cdot 3_{4}(\operatorname{ArC}), 132 \cdot 0\left(d,{ }^{5} J(\mathrm{P}-\right.$ $\mathrm{C})=5.0 \mathrm{~Hz}, \quad \mathrm{CH}=\mathrm{CH}(\mathrm{Me})), \quad 212.8 \quad\left(d, \quad{ }^{2} \mathrm{~J}(\mathrm{P}-\right.$ $\mathrm{C})=3.0 \mathrm{~Hz}, \mathrm{PCC}) .{ }^{31} \mathrm{P}$ NMR $\left(160 \mathrm{MHz}, \mathrm{CDCl}_{3}\right): \delta$ 6.7. Anal. Calc. for $\mathrm{C}_{17} \mathrm{H}_{21} \mathrm{O}_{3} \mathrm{P}: \mathrm{C}, 67.09 ; \mathrm{H}, 6.96$. Found: C, 67.05; H, 6.96 .

\subsection{Synthesis of the phosphonate allenol} $\left.\mathrm{C}\left(\mathrm{CH}_{2} \mathrm{O}\right)_{2}\left\{\mathrm{P}(\mathrm{O}) \mathrm{C}_{2} \mathrm{CH}_{2}(\mathrm{OH})\right]=\mathrm{C}=\mathrm{CH}_{2}\right\}$ (6) and the phosphono-butadiene $\left[\left(\mathrm{OCH}_{2} \mathrm{CMe}_{2} \mathrm{CH}_{2} \mathrm{O}\right)(\mathrm{O})\right.$ $\left.\mathrm{P}-\mathrm{C}\left(=\mathrm{CH}_{2}\right)\right]_{2}$ (7)

To a solution of diol $(4.60 \mathrm{~g}, 53.4 \mathrm{mmol})$ and triethylamine $(5.41 \mathrm{~g}, 7.45 \mathrm{~mL}, 53.4 \mathrm{mmol})$ in THF $(135 \mathrm{~mL})$, compound $1 \quad(9.00 \mathrm{~g}, \quad 7.40 \mathrm{~mL}$, $53.4 \mathrm{mmol})$ in THF $(25 \mathrm{~mL})$ was added drop-wise at $-78^{\circ} \mathrm{C}$, the mixture stirred for $2 \mathrm{~h}$, brought to room temperature and was then heated under reflux for 15-18 h. Triethylamine hydrochloride formed was filtered off and the solvent removed in vacuo. Yield was $80 \%$ on the basis of ${ }^{31} \mathrm{P}$ NMR; the remaining material was the 1,3-butadiene $\left[\left(\mathrm{OCH}_{2} \mathrm{CMe}_{2} \mathrm{CH}_{2} \mathrm{O}\right)\right.$ (O) $\left.\mathrm{P}-\mathrm{C}\left(=\mathrm{CH}_{2}\right)\right]_{2}(7)$. Pure compound 6 [isolated yield $2.0 \mathrm{~g}(17 \%)$; low yield because of the other product] was obtained by crystallization from THF. M.p.: $134-138^{\circ} \mathrm{C}$. IR (KBr): 3304, 3069, 2975, 2938, 1968, 1939, 1827, 1719, 1472, 1238, 1061, $1009,839 \mathrm{~cm}^{-1} .{ }^{1} \mathrm{H}$ NMR: 0.99 and $1.19(2 s, 6 \mathrm{H}$, $\left.\mathrm{C}\left(\mathrm{CH}_{3}\right)_{2}\right), 2.50(\mathrm{br}, 1 \mathrm{H}, \mathrm{OH}), 4 \cdot 00-4 \cdot 12(\mathrm{~m}, 4 \mathrm{H}$, $\left.\mathrm{OCH}_{2}\right), \quad 4.33 \quad\left(t d,{ }^{3} J(\mathrm{P}-\mathrm{H})=14.0 \mathrm{~Hz},{ }^{5} J(\mathrm{H}-\mathrm{H})=\right.$ $\left.2.0 \mathrm{~Hz}, 2 \mathrm{H}, \mathrm{CH}_{2} \mathrm{OH}\right), 5.15\left(t d,{ }^{4} \mathrm{~J}(\mathrm{P}-\mathrm{H})=12.8 \mathrm{~Hz}\right.$, $\left.{ }^{5} J(\mathrm{H}-\mathrm{H})=2.0 \mathrm{~Hz}, 2 \mathrm{H}, \mathrm{C}=\mathrm{CH}_{2}\right) \cdot{ }^{13} \mathrm{C} \mathrm{NMR}: 20.9$ and $21.7 \quad\left(2 \mathrm{~s}, \quad \mathrm{C}\left(\mathrm{CH}_{3}\right)_{2}\right), \quad 32.5 \quad\left(d,{ }^{3} J(\mathrm{P}-\mathrm{C})=7.0 \mathrm{~Hz}\right.$, $\left.C\left(\mathrm{CH}_{3}\right)_{2}\right), 60 \cdot 3\left(d,{ }^{2} J(\mathrm{P}-\mathrm{C})=8 \cdot 0 \mathrm{~Hz}, \mathrm{CH}_{2} \mathrm{OH}\right), 77 \cdot 1$ $\left(d,{ }^{2} J(\mathrm{P}-\mathrm{C})=6.0 \mathrm{~Hz}, \quad \mathrm{POCH}_{2}\right), \quad 78.0 \quad\left(d,{ }^{3} J(\mathrm{P}-\right.$ $\left.\mathrm{C})=14.0 \mathrm{~Hz}, \mathrm{C}=\mathrm{CH}_{2}\right), 92.9\left(d,{ }^{1} J(\mathrm{P}-\mathrm{C})=183.0 \mathrm{~Hz}\right.$, $\mathrm{PC}), 211.3\left(d,{ }^{2} J(\mathrm{P}-\mathrm{C})=6.0 \mathrm{~Hz}, \mathrm{PC}=C\right) .{ }^{31} \mathrm{P}$ NMR: 10.1. Anal. Calcd for $\mathrm{C}_{9} \mathrm{H}_{15} \mathrm{O}_{4}$ : C: $49.54, \mathrm{H} 6.93$. Found: C, 49.47; H, 7.02.

Compound 7: This was isolated from the same reaction in low yield $(0.93 \mathrm{~g}, 5 \%)$, but a quantitative yield could be obtained by using $1: 2$ molar reaction of propargylic alcohol to 1. M.p.: $182-186^{\circ} \mathrm{C}$; IR (KBr): 2973, 2888, 1931, 1578, 1478, 1265, 1055, $1005,957 \mathrm{~cm}^{-1}$. ${ }^{1} \mathrm{H}$ NMR: $\delta 0.96$ and $1.24(2 s, 12 \mathrm{H}$, $\left.\mathrm{C}\left(\mathrm{CH}_{3}\right)_{2}\right), 3.98-4 \cdot 11\left(\mathrm{~m}, 8 \mathrm{H}, \mathrm{OCH}_{2}\right), 6 \cdot 27\left(d,{ }^{3} \mathrm{~J}(\mathrm{P}-\right.$ $\mathrm{H})=20.1 \mathrm{~Hz}, 1 \mathrm{H},=\mathrm{CH}_{\mathrm{A}} \mathrm{H}_{\mathrm{B}}$, cis to $\left.\mathrm{P}\right), 6.56\left(d,{ }^{3} J(\mathrm{P}-\right.$ $\mathrm{H})=44.9 \mathrm{~Hz}, 1 \mathrm{H},=\mathrm{CH}_{\mathrm{A}} H_{\mathrm{B}}$, trans to $\left.\mathrm{P}\right){ }^{13} \mathrm{C}$ NMR: $\delta$ $21 \cdot 1,21.9\left(2 s, \mathrm{C}\left(\mathrm{CH}_{3}\right)_{2}\right), 32.5$ (d with virtual coupling (?), $\left.{ }^{3} J(\mathrm{P}-\mathrm{C}) \sim 6.0 \mathrm{~Hz}, C \mathrm{Me}_{2}\right), 76.9$ (d with virtual coupling (?), $\left.{ }^{2} J(\mathrm{PC}) \sim 6 \cdot 0 \mathrm{~Hz}, \mathrm{OCH}_{2}\right), 132 \cdot 1(d$, $\left.{ }^{1} J(\mathrm{P}-\mathrm{C})=182 \cdot 0 \mathrm{~Hz}, \mathrm{PC}\right), 134.2(d$ with virtual coupling (?), $\left.{ }^{2} J(\mathrm{P}-\mathrm{C}) \sim 12 \cdot 0 \mathrm{~Hz}, \mathrm{PC}=C\right) \cdot{ }^{31} \mathrm{P}$ NMR: $\delta$ 9.0. Anal. Calcd for $\mathrm{C}_{14} \mathrm{H}_{24} \mathrm{O}_{6} \mathrm{P}_{2}$ : C: 49.54, H 6.93. Found: C, 47.97; H, 6.93.

\subsection{Synthesis of bis(allenyl)phosphonates}

$\left[\mathrm{R}^{1} \mathrm{R}^{2} \mathrm{C}=\mathrm{C}=\mathrm{C}(\mathrm{H}) \mathrm{P}(\mathrm{O})\left(\mathrm{OCH}_{2}\right)_{2}\right]_{2} \mathrm{C}\left(\mathrm{R}^{1}=\mathrm{R}^{2}=\mathrm{H}(9)\right.$, $\left.R^{1}=M e, R^{2}=E t(10), R^{1}=R^{2}=M e(11)\right):$ Typical procedure for 9 and identification of the intermediate 8

To a solution of $2(2.04 \mathrm{~g}, 7.4 \mathrm{mmol})$ and triethylamine $(1.49 \mathrm{~g}, 14.8 \mathrm{mmol})$ in dry THF $(30 \mathrm{~mL})$ at $0{ }^{\circ} \mathrm{C}$, the propargyl alcohol $(14.8 \mathrm{mmol})$ was added at $0^{\circ} \mathrm{C}$ with continuous stirring. The reaction mixture was brought to room temperature, stirred for $3 \mathrm{~h}$ and filtered. The tricoordinate compound $8\left[>90 \%\right.$ by ${ }^{31} \mathrm{P}$ NMR] was characterized by ${ }^{1} \mathrm{H}$ and ${ }^{31} \mathrm{P}$ NMR spectra [ ${ }^{1} \mathrm{H}$ NMR: $2.43(s, 2 \mathrm{H}, \mathrm{C} \equiv \mathrm{CH}), 3.30-3.35$ and 3.92- 
3.96 ( $\mathrm{m}$ each, $2+2 \mathrm{H}, \mathrm{OCH}_{2}-\mathrm{C} \equiv \mathrm{CH}$; the two groups are in slightly different environments), 4.20-4.46 ( $\left.m, 8 \mathrm{H}, \mathrm{CH}_{2} \mathrm{OP}\right) .{ }^{31} \mathrm{P}$ NMR: $\left.125 \cdot 7\right]$. In the ${ }^{1} \mathrm{H}$ NMR spectrum taken at this stage, solvent peaks (THF: $\delta$ 1.80 and 3.70 ) were also observed. The filtrate was heated under reflux for $15-18 \mathrm{~h}$ and the solvent removed in vacuo. Compound 9 (1.72 g, 79\%) was purified by crystallization from methanol. M.p.: 199$201^{\circ} \mathrm{C}$. IR (KBr): 3069, 2984, 2915, 1944, 1474, $1279,1237,1194,1156,1024,843 \mathrm{~cm}^{-1}$. ${ }^{1} \mathrm{H}$ NMR: $4 \cdot 17-4.27$ and $4.42-4.52\left(2 \mathrm{~m}, 8 \mathrm{H}, \mathrm{OCH}_{2}\right), 5 \cdot 16-$ $5.21\left(m, 4 \mathrm{H}, \mathrm{C}=\mathrm{CH}_{2}\right), 5.40-5.42(m, 2 \mathrm{H}, \mathrm{PC} H)$. The compound had a poor solubility and hence ${ }^{13} \mathrm{C}$ NMR spectrum was not recorded. ${ }^{31} \mathrm{P}$ NMR: 11.36. Anal. Calcd. $\mathrm{C}_{11} \mathrm{H}_{14} \mathrm{O}_{6} \mathrm{P}_{2}$ : C, 43.44; H, 4.64. Found: C, 43.42; H, 4.69.

Compounds 10 and 11 were purified using column chromatography (ethyl acetate). Compound $\mathbf{1 2}$ was purified by column chromatography using hexane-ethyl acetate $(1: 1)$ mixture. Yields were above $95 \%$ for all these compounds on the basis of crude ${ }^{31} \mathrm{P}$ NMR using the same molar quantities; isolated yields are given below.

2.3a $\mathrm{C}\left(\mathrm{CH}_{2} \mathrm{O}\right)_{4}\left\{\mathrm{P}(\mathrm{O}) \mathrm{C}(\mathrm{H})=\mathrm{C}=\mathrm{CMeEt}_{2}(10)\right.$ : Yield: $2.11 \mathrm{~g}, 74 \%$. M.p.: $106-110^{\circ} \mathrm{C}$. IR (KBr): 2972 , $2915,1962,1698,1458,1370,1269,1200,1150$, $1020,828 \mathrm{~cm}^{-1} .{ }^{1} \mathrm{H}$ NMR: $\delta \quad 1.07 \quad\left(t,{ }^{3} J(\mathrm{H}-\right.$ $\left.\mathrm{H})=7.2 \mathrm{~Hz}, \quad 6 \mathrm{H}, \quad \mathrm{CH}_{2} \mathrm{CH}_{3}\right), \quad 1.80-1.83 \quad(m, 6 \mathrm{H}$, $\left.\mathrm{C}=\mathrm{CCH}_{3}\right), 2 \cdot 04-2 \cdot 13\left(m, 4 \mathrm{H}, \mathrm{CH}_{2} \mathrm{CH}_{3}\right), 4 \cdot 16-4.48$ $\left(m, 8 \mathrm{H}, \mathrm{OCH}_{2}\right), 5 \cdot 30-5 \cdot 35(m, 2 \mathrm{H}, \mathrm{PCH}) .{ }^{13} \mathrm{C} \mathrm{NMR}$ : $\delta 11.9\left(s, \mathrm{CH}_{2} \mathrm{CH}_{3}\right), 17.5$ and $17.6\left(2 s, \mathrm{CH}_{2} \mathrm{CH}_{3}\right)$, 26.0 and $26 \cdot 1 \quad\left(2 s, \quad \mathrm{C}=\mathrm{CCH}_{3}\right), \quad 37.3 \quad\left(t,{ }^{3} J(\mathrm{P}-\right.$ C) $\left.=6.0 \mathrm{~Hz}, \mathrm{OCH}_{2} C\right), 67.5,67.6,67.7$ and $67.9(4 d$, $\left.{ }^{2} J(\mathrm{P}-\mathrm{C}) \sim 6.0 \mathrm{~Hz}, \quad \mathrm{OCH}_{2}\right), \quad 77.3 \quad\left(d, \quad{ }^{1} J(\mathrm{P}-\mathrm{C})=\right.$ $199.1 \mathrm{~Hz}, \quad \mathrm{PC}), \quad 104.5 \quad\left(d,{ }^{3} J(\mathrm{P}-\mathrm{C})=17.4 \mathrm{~Hz}, \mathrm{P}-\right.$ $\mathrm{C}=\mathrm{C}=C(\mathrm{Me})(\mathrm{Et})), \quad 211.8(s, \mathrm{PC}=C) .{ }^{31} \mathrm{P}$ NMR: $\delta$ 13.0. LC/MS $m / z 389[\mathrm{M}+1]^{+}$. Diastereomers may be expected in this case, but we did not get evidence for the presence of these in our studies.

2.3b $\quad C\left(\mathrm{CH}_{2} \mathrm{O}\right)_{4}\left\{\mathrm{P}(\mathrm{O}) \mathrm{C}(\mathrm{H})=\mathrm{C}=\mathrm{CMe}_{2}\right\}_{2}$ (11): Yield: 1.98 g, $76 \%$. M.p.: $140-144^{\circ} \mathrm{C}$. IR (KBr): 2986, 2907, 1964, 1682, 1364, 1269, 1200, 1152, 1020, $820 \mathrm{~cm}^{-1} .{ }^{1} \mathrm{H} \quad$ NMR: $\delta \quad 1.80-1.88 \quad(m, 12 \mathrm{H}$, $\left.\mathrm{C}=\mathrm{C}\left(\mathrm{CH}_{3}\right)_{2}\right), 4.09-4.50\left(m, 8 \mathrm{H}, \mathrm{OCH}_{2}\right), 5 \cdot 22-5.25 \mathrm{~m}$, $2 \mathrm{H}, \quad \mathrm{PC} H) .{ }^{13} \mathrm{C}$ NMR: $\delta \quad 19.0$ and $19.1 \quad(2 s$, $\left.\mathrm{C}=\mathrm{C}\left(\mathrm{CH}_{3}\right)_{2}\right), 37.2\left(s, \mathrm{OCH}_{2} \mathrm{C}\right), 67.7$ and $67.9(2 d$, $\left.{ }^{2} J(\mathrm{P}-\mathrm{C})=6.5 \mathrm{~Hz}, \mathrm{OCH}_{2}\right), 75.4\left(d,{ }^{1} J(\mathrm{P}-\mathrm{C})=199.1 \mathrm{~Hz}\right.$, PC $), 98.6\left(d,{ }^{3} J(\mathrm{P}-\mathrm{C})=17.2 \mathrm{~Hz}, \mathrm{C}=C \mathrm{Me}_{2}\right), 212.3$ $(s, \mathrm{PC}=C) .{ }^{31} \mathrm{P}$ NMR: $\delta$ 12.6. LC/MS $m / z \quad 361$ $[\mathrm{M}+1]^{+}$.
Allenylphosphonates 12-16 Compounds 12 and 14 were prepared by using literature procedures. ${ }^{10}$ Allenes $\mathbf{1 3}$ and $\mathbf{1 5}-\mathbf{1 6}$ were synthesized similarly using $10 \cdot 0 \mathrm{mmol}$ of 1 .

$2.3 \mathrm{c} \quad\left(\mathrm{OCH}_{2} \mathrm{CMe}_{2} \mathrm{CH}_{2} \mathrm{O}\right) \mathrm{P}(\mathrm{O}) \mathrm{CH}=\mathrm{C}=\mathrm{CMe}(\mathrm{H})$ (13): Yield: $1.74 \mathrm{~g}(86 \%)$. M.p.: $50-52^{\circ} \mathrm{C}$. IR (KBr): 1956 , $1476,1373,1281,1061,1011 \mathrm{~cm}^{-1}$. ${ }^{1} \mathrm{H}$ NMR: $\delta 0.94$ and $1.16\left(2 s, 6 \mathrm{H}, \mathrm{C}\left(\mathrm{CH}_{3}\right)_{2}\right), 1.68-1.82(m, 3 \mathrm{H}$, $\left.\mathrm{C}=\mathrm{C}(\mathrm{H}) \mathrm{CH}_{3}\right), 3 \cdot 96-4 \cdot 12\left(m, 4 \mathrm{H}, \mathrm{OCH}_{2}\right), 5 \cdot 24-5 \cdot 32$ ( $m, 1 \mathrm{H}, \mathrm{CHMe}), 5.33-5.59(m, 1 \mathrm{H}, \mathrm{PC} H) .{ }^{13} \mathrm{C} \mathrm{NMR}$ : $\delta 12.3\left(d,{ }^{4} J(\mathrm{PC})=7.0 \mathrm{~Hz}, \mathrm{CHCH}_{3}\right), 20.4$ and 21.3 $\left(2 s, \mathrm{C}\left(\mathrm{CH}_{3}\right)_{2}\right), 32 \cdot 1\left(d,{ }^{3} J(\mathrm{PC})=6 \cdot 0 \mathrm{~Hz}, C \mathrm{Me}_{2}\right), 76 \cdot 4$ and $76.5\left(2 d,{ }^{2} J(\mathrm{PC})=6.0 \mathrm{~Hz}\right.$ each, $\left.\mathrm{OCH}_{2}\right), 77.5(d$, $\left.{ }^{1} J(\mathrm{PC})=192.0 \mathrm{~Hz}, \mathrm{PC}\right), 86.9\left(d,{ }^{3} J(\mathrm{PC})=16.0 \mathrm{~Hz}\right.$, $\left.\mathrm{C}=\mathrm{CH}_{2}\right), 212 \cdot 3($ br s, $\mathrm{C}=C=\mathrm{CHMe}) .{ }^{31} \mathrm{P}$ NMR: $\delta 7.9$.

$2.3 \mathrm{~d} \quad\left(\mathrm{OCH}_{2} \mathrm{CMe}_{2} \mathrm{CH}_{2} \mathrm{O}\right) \mathrm{P}(\mathrm{O}) \mathrm{CH}=\mathrm{C}=\mathrm{CMeEt} \quad$ (15): Yield: $1.79 \mathrm{~g}(78 \%)$. M.p.: $93-94^{\circ} \mathrm{C}$. IR (KBr): 1958 , 1474, 1372, 1277, 1061, $1009 \mathrm{~cm}^{-1} .{ }^{1} \mathrm{H}$ NMR: $\delta 0 \cdot 83$ and $1.04\left(2 s, 6 \mathrm{H}, \mathrm{C}\left(\mathrm{CH}_{3}\right)_{2}\right), 0.92\left(t,{ }^{3} J(\mathrm{HH})=7.8 \mathrm{~Hz}\right.$, $\left.3 \mathrm{H}, \quad \mathrm{C}=\mathrm{CMeCH}_{2} \mathrm{CH}_{3}\right), \quad 1.65 \quad\left(d d,{ }^{5} J(\mathrm{PH})=6.8 \mathrm{~Hz}\right.$, $\left.{ }^{5} J(\mathrm{HH})=2.9 \mathrm{~Hz}, 3 \mathrm{H}, \mathrm{C}=\mathrm{CCH}_{3} \mathrm{Et}\right), 1.88-1.97(m, 2 \mathrm{H}$, $\left.\mathrm{C}=\mathrm{CMeCH}_{2} \mathrm{CH}_{3}\right), 3.79-3.96\left(m, 4 \mathrm{H}, \mathrm{OCH}_{2}\right), 5 \cdot 14-$ $5.19(m, 1 \mathrm{H}, \mathrm{PC} H) .{ }^{13} \mathrm{C}$ NMR: $\delta 11.7\left(d,{ }^{5} \mathrm{~J}(\mathrm{PC})=\right.$ $\left.2.4 \mathrm{~Hz}, \mathrm{C}=\mathrm{CMeCH}_{2} \mathrm{CH}_{3}\right), 17.5\left(d,{ }^{4} J(\mathrm{PC})=7.3 \mathrm{~Hz}\right.$, $\left.=\mathrm{CMeCH}_{2} \mathrm{CH}_{3}\right), 20.8$ and $21.5\left(2 \mathrm{~s}, \mathrm{C}\left(\mathrm{CH}_{3}\right)_{2}\right), 26.0(d$, $\left.{ }^{4} J(\mathrm{PC})=7.3 \mathrm{~Hz}, \mathrm{CCH}_{3} \mathrm{Et}\right), 32.3\left(d,{ }^{3} J(\mathrm{PC})=6 \cdot 1 \mathrm{~Hz}\right.$, $\left.C \mathrm{Me}_{2}\right), 77.9\left(2 d,{ }^{1} J(\mathrm{PC})=195.3 \mathrm{~Hz}, \mathrm{PC}\right), 76.2$ and $76 \cdot 3\left(2 s, \mathrm{OCH}_{2}\right), 103 \cdot 3\left(d,{ }^{3} J(\mathrm{PC})=17 \cdot 0 \mathrm{~Hz}, \mathrm{C}=\right.$ $C \mathrm{MeEt}$ ), 210.3 (br s, $\mathrm{C}=C=\mathrm{CMeEt}$ ). ${ }^{31} \mathrm{P}$ NMR: $\delta 9 \cdot 3$. Anal. Calcd for $\mathrm{C}_{11} \mathrm{H}_{19} \mathrm{O}_{3} \mathrm{P}: \mathrm{C}, 57 \cdot 39 ; \mathrm{H}, 8 \cdot 26$. Found: C, 57.64; H, 8.13.

$2.3 \mathrm{e} \quad\left(\mathrm{OCH}_{2} \mathrm{CMe}_{2} \mathrm{CH}_{2} \mathrm{O}\right) \mathrm{P}(\mathrm{O}) \mathrm{C}(\mathrm{Ph})=\mathrm{C}=\mathrm{C}(\mathrm{H}) \mathrm{Et}(\mathbf{1 6})$ : Yield: $2.45 \mathrm{~g}(84 \%)$. M.p.: $108-109^{\circ} \mathrm{C}$. IR (KBr): $3057,3022,1948,1597,1493,1372,1262,1059$, $1009 \mathrm{~cm}^{-1} .{ }^{1} \mathrm{H}$ NMR: $\delta \quad 0.83$ and $1.23(2 s, 6 \mathrm{H}$, $\left.\mathrm{C}\left(\mathrm{CH}_{3}\right)_{2}\right), 1.09\left(t,{ }^{3} \mathrm{~J}(\mathrm{HH})=7.6 \mathrm{~Hz}, 3 \mathrm{H}, \mathrm{CH}_{2} \mathrm{CH}_{3}\right)$, $2 \cdot 18\left(q r t,{ }^{3} J(\mathrm{HH})=7 \cdot 6 \mathrm{~Hz}, 2 \mathrm{H}, \mathrm{CH}_{2} \mathrm{CH}_{3}\right), 3 \cdot 77-4.01$ $\left(m, 4 \mathrm{H}, \mathrm{OCH}_{2}\right), 5 \cdot 70-5.89(m, 1 \mathrm{H}, \mathrm{PCH}), 7 \cdot 17-7 \cdot 28$ $\left(m, 3 \mathrm{H}, \mathrm{Ar}-H\right.$ (meta and para)), $7.55\left(d,{ }^{3} J(\mathrm{HH}) \sim\right.$ $7.0 \mathrm{~Hz}, \mathrm{Ar}-H$ (ortho)). ${ }^{13} \mathrm{C}$ NMR: $\delta 13.4\left(s, \mathrm{CH}_{2} \mathrm{CH}_{3}\right)$, $20 \cdot 8\left(s, \mathrm{CH}_{3}\right), 21.4\left(d,{ }^{4} \mathrm{~J}(\mathrm{PC})=6 \cdot 1 \mathrm{~Hz}, \mathrm{CH}_{2} \mathrm{CH}_{3}\right)$, $21.9\left(s, \mathrm{CH}_{3}\right), 32.5\left(d,{ }^{3} J(\mathrm{PC})=6.6 \mathrm{~Hz}, C \mathrm{Me}_{2}\right), 76.8$ and $76.9\left(2 s, \mathrm{OCH}_{2}\right), 96.4\left(d,{ }^{1} J(\mathrm{PC}) \sim 185.0 \mathrm{~Hz}\right.$, $\mathrm{PC}), 96.8\left(d,{ }^{3} J(\mathrm{PC})=14.9 \mathrm{~Hz}, \mathrm{C}=C \mathrm{HEt}\right), 127.5$, $127.6,127.7,128.6,132.3,132.4,209.5$ (br s, $\mathrm{C}=C=\mathrm{CHEt}) .{ }^{31} \mathrm{P}$ NMR: $\delta 7 \cdot 3$.

2.3f $\left[\mathrm{H}_{2} \mathrm{C}=\mathrm{C}=\mathrm{C}(\mathrm{Ph}) \mathrm{P}(\mathrm{O})\left(\mathrm{OCH}_{2}\right)_{2}\right]_{2} \mathrm{C} \quad$ (17): This compound was prepared in a manner similar to that for 9 using the same molar quantities. Yield: $2.59 \mathrm{~g}$ 
(74\%). M.p. $>300^{\circ} \mathrm{C}$. IR (KBr): 3059, 2982, 1939 , $1595,1493,1269,1155,1076,1020,938 \mathrm{~cm}^{-1} .{ }^{1} \mathrm{H}$ NMR $\left(400 \mathrm{MHz}, \mathrm{CDCl}_{3}\right): \delta 3.99-4.04,4.22-4.33$ and 4.58-4.65 (three groups of $m, 8 \mathrm{H}, \mathrm{OCH}_{2}$ ), 5.42 $\left(d, 4 \mathrm{H},{ }^{4} J(\mathrm{P}-\mathrm{H})=13.2 \mathrm{~Hz},=\mathrm{CH}_{2}\right), 7.26-7.55(\mathrm{~m}$, $10 \mathrm{H}, \mathrm{Ar} H) ;{ }^{13} \mathrm{C}$ NMR $\left(100 \mathrm{MHz}, \mathrm{CDCl}_{3}\right): \delta 37.5(t$, $\left.{ }^{3} J(\mathrm{P}-\mathrm{C})=5.7 \mathrm{~Hz}, \mathrm{OCH}_{2} C\right), 68.5$ and $68.9\left(2 d,{ }^{2} J(\mathrm{P}-\right.$ $\mathrm{C})=7.0 \mathrm{~Hz}$ each, $\left.\mathrm{OCH}_{2}\right), 79.5\left(d,{ }^{3} J(\mathrm{P}-\mathrm{C})=15.1 \mathrm{~Hz}\right.$, $\left.\mathrm{C}=\mathrm{CH}_{2}\right), 94.8\left(d,{ }^{1} J(\mathrm{P}-\mathrm{C})=185.2 \mathrm{~Hz}, \mathrm{PC}\right), 127.7$, $127.8,128.5,129.0,129.9,130.0(\operatorname{ArC}), 213.5(d$, $\left.{ }^{2} J(\mathrm{P}-\mathrm{C}) \sim 4.6 \mathrm{~Hz}, \mathrm{C}=\mathrm{C}=\mathrm{C}\right) .{ }^{31} \mathrm{P}$ NMR $(160 \mathrm{MHz}$, $\left.\mathrm{CDCl}_{3}\right): \delta 10 \cdot 6 . \mathrm{LC} / \mathrm{MS} m / z 457[\mathrm{M}+1]^{+}$.

2.4 Synthesis of the allene dimer $\left[\mathrm{COCH}_{2} \mathrm{C}\right.$ $\left.\left.\mathrm{Me}_{2} \mathrm{CH}_{2} \mathrm{O}\right) \mathrm{PCH}=\right]_{2}\left[\mathrm{C}-\mathrm{CMe}_{2}\right]_{2}(18):$ Allene $14(0.2 \mathrm{~g}$, $0.92 \mathrm{mmol})$ was heated at $185^{\circ} \mathrm{C}$ for $6 \mathrm{~h}$. TLC (EtOAc/hexane) showed two spots (approximate ratio $4:$ 1) under the UV lamp. The major component (18) was separated by column chromatography (silica gel, ethyl acetate-hexane, $3: 2$ ). Yield (isolated): $0.14 \mathrm{~g}$ (68\%). M.p.: $226-228^{\circ} \mathrm{C}$. IR (KBr): 1622, 1476, $1372,1265,1061,1013 \mathrm{~cm}^{-1}$. ${ }^{1} \mathrm{H}$ NMR $(400 \mathrm{MHz}$, $\left.\mathrm{CDCl}_{3}\right): \delta 1.07$ and $1.10\left(2 s, 12 \mathrm{H}, \mathrm{C}\left(\mathrm{CH}_{3}\right)_{2}\right), 1.33(s$, $\left.12 \mathrm{H},=\mathrm{C}\left(\mathrm{CH}_{3}\right)_{2}\right), \quad 3.87\left(d d \rightarrow t,{ }^{3} \mathrm{~J}(\mathrm{P}-\mathrm{H}) \sim 11.3 \mathrm{~Hz}\right.$ each, $\left.2 \mathrm{H}, \mathrm{OCH}_{2}\right), 4 \cdot 15\left(d d \rightarrow t,{ }^{3} J(\mathrm{P}-\mathrm{H}) \sim 11.3 \mathrm{~Hz}\right.$ each, $\left.2 \mathrm{H}, \mathrm{OCH}_{2}\right), 5.62\left(d,{ }^{2} J(\mathrm{P}-\mathrm{H})=16.3 \mathrm{~Hz}, 1 \mathrm{H}\right.$, $\mathrm{PCH}) .{ }^{13} \mathrm{C}$ NMR $\left(50 \mathrm{MHz}, \mathrm{CDCl}_{3}\right): \delta 21.5$ and 22.1 (2s, $\mathrm{CH}\left(\mathrm{CH}_{3}\right)_{2}+=\mathrm{C}\left(\mathrm{CH}_{3}\right)_{2}$, two methyl signals have merged), 32.6 (br, CMe $\mathrm{Me}_{2}$ ), 49.7 ( $b r, C \mathrm{Me}_{2}$ of four membered ring), $75.6 \quad\left(\mathrm{OCH}_{2}\right), 105.2\left(d,{ }^{1} J(\mathrm{P}-\right.$ C) $=186.8 \mathrm{~Hz}, \mathrm{PCH}), 170.8\left(d,{ }^{2} J(P-C)=43.5 \mathrm{~Hz}\right.$, $\mathrm{PC}=C) .{ }^{31} \mathrm{P}$ NMR $\left(160 \mathrm{MHz}, \mathrm{CDCl}_{3}\right): \delta 11 \cdot 0 . \mathrm{LC}-$ MS: $m / z 434[\mathrm{M}+2]^{+}$. Anal. Calcd. for $\mathrm{C}_{20} \mathrm{H}_{34} \mathrm{O}_{6} \mathrm{P}_{2}$ : C, 55.54; H, 7.92. Found: C, 55.47; H, 7.97.

This reaction was also performed in the presence of hydroquinone (radical quencher), but the ${ }^{31} \mathrm{P}$ NMR spectrum of the reaction mixture showed several products $[\delta(P):-12 \cdot 3,10 \cdot 1,10 \cdot 2,11 \cdot 0,11 \cdot 3$, $13 \cdot 3,21 \cdot 8,23 \cdot 9]$ and hence was not analysed further.

2.4a Attempted synthesis of the anthracene adduct $\left(\mathrm{OCH}_{2} \mathrm{CMe}_{2} \mathrm{CH}_{2} \mathrm{O}\right) \mathrm{PCH}\left(\mathrm{C}_{14} \mathrm{H}_{10}\right) \quad \mathrm{C}=\mathrm{CMe}_{2}$ (19): A mixture of allene $14(0.9 \mathrm{~g}, 4.16 \mathrm{mmol})$ and anthracene $(1.13 \mathrm{~g}, 6.30 \mathrm{mmol})$ was heated at $185^{\circ} \mathrm{C}$ for 5 $6 \mathrm{~h}$. TLC (EtOAc/hexane) showed a new UV active spot under the UV lamp and some unreacted starting material 14. The compound corresponding to the UV active spot (18) was separated by column chromatography [silica gel, ethyl acetate-hexane, $3: 2$; yield $0.36 \mathrm{~g} \mathrm{(20 \% )].} \mathrm{X-ray} \mathrm{structure} \mathrm{was} \mathrm{determined} \mathrm{for} \mathrm{this}$ sample. The expected compound 19 could not be identified.
2.5 Synthesis of the enaminophosphonates $\left(\mathrm{OCH}_{2} \mathrm{CMe}_{2} \mathrm{CH}_{2} \mathrm{O}\right) \mathrm{P}(\mathrm{O}) \mathrm{CH}=\mathrm{C}\left(\mathrm{NEt}_{2}\right) \mathrm{CH}_{2} \mathrm{R}$ [R=H (20), Me (21)]

To a solution of allenylphosphonates (12-13) $(1.0 \mathrm{mmol})$ in acetonitrile $(15 \mathrm{~mL})$, diethylamine $(1.0 \mathrm{mmol})$ was added slowly via syringe. The reaction mixture was heated under reflux for $4 \mathrm{~h}$ with continuous stirring. Solvent was removed in vacuo to obtain the required compound. The reaction was very clean without any side products. These were characterized spectroscopically, because of their hydrolytic instability leading to the $\beta$-ketophosphonates.

2.5a Compound 20: Yield: Quantitative. M.p.: 58$60^{\circ} \mathrm{C}$. IR (KBr): $1570,1439,1219,1061,1001 \mathrm{~cm}^{-1}$. ${ }^{1} \mathrm{H}$ NMR: $\delta 0.92$ and $1.10\left(2 s, 6 \mathrm{H}, \mathrm{C}\left(\mathrm{CH}_{3}\right)_{2}\right), 1.09(t$, $\left.{ }^{3} J(\mathrm{H}-\mathrm{H})=7 \cdot 1 \mathrm{~Hz}, 3 \mathrm{H}, \mathrm{N}\left(\mathrm{CH}_{2} \mathrm{CH}_{3}\right)_{2}\right), 2.26\left(d,{ }^{4} J(\mathrm{P}-\right.$ $\left.\mathrm{H})=1.5 \mathrm{~Hz}, \quad 3 \mathrm{H}, \quad \mathrm{C}\left(\mathrm{NEt}_{2}\right) \mathrm{CH}_{3}\right), 3.20 \quad\left(q,{ }^{3} J(\mathrm{H}-\right.$ $\left.\mathrm{H})=7 \cdot 1 \mathrm{~Hz}, 2 \mathrm{H}, \mathrm{N}\left(\mathrm{CH}_{2} \mathrm{CH}_{3}\right)_{2}\right), 3.65-3.74(m, 3 \mathrm{H}$, $\left.\mathrm{OCH}_{2}+\mathrm{PCH}\right), \quad 4 \cdot 16-4 \cdot 20 \quad\left(d d \rightarrow t, \quad{ }^{2} J(\mathrm{H}-\mathrm{H})=\right.$ $\left.2.3 \mathrm{~Hz},{ }^{3} \mathrm{~J}(\mathrm{P}-\mathrm{H}) \sim 9.4 \mathrm{~Hz}, 2 \mathrm{H}, \mathrm{OCH}_{2}\right) .{ }^{13} \mathrm{C} \mathrm{NMR}: \delta$ $12.4 \quad\left(s, \quad \mathrm{C}\left(\mathrm{NCH}_{2} \mathrm{CH}_{3}\right)_{2} \mathrm{Me}\right), \quad 17.2 \quad\left(d,{ }^{3} J(\mathrm{P}-\mathrm{C})=\right.$ $\left.4.4 \mathrm{~Hz}, \mathrm{C}\left(\mathrm{NEt}_{2}\right) \mathrm{CH}_{3}\right), 21.2$ and $21.6\left(2 \mathrm{~s}, \mathrm{C}\left(\mathrm{CH}_{3}\right)_{2}\right)$, $32.1\left(d,{ }^{3} J(\mathrm{P}-\mathrm{C})=4.8 \mathrm{~Hz}, C \mathrm{Me}_{2}\right), 43.6\left(s, \mathrm{C}\left(\mathrm{NCH}_{2}\right.\right.$ $\left.\left.\mathrm{CH}_{3}\right)_{2} \mathrm{Me}\right), 70 \cdot 1\left(d,{ }^{1} J(\mathrm{P}-\mathrm{C})=218.9 \mathrm{~Hz}, \mathrm{PC}\right), 74 \cdot 3$, $74.4 \quad\left(2 s, \quad \mathrm{OCH}_{2}\right), \quad 160.0 \quad\left(d,{ }^{2} J(\mathrm{P}-\mathrm{C})=21.6 \mathrm{~Hz}\right.$, $\mathrm{PCH}=C) .{ }^{31} \mathrm{P}$ NMR: $\delta 24 \cdot 7$.

2.5b Compound 21: Yield: Quantitative. M.p.: $55-56^{\circ} \mathrm{C}$. IR (KBr): 1573, 1474, 1360, 1217, 1059, $1005 \mathrm{~cm}^{-1} .{ }^{1} \mathrm{H}$ NMR: $\delta 0.84\left(\mathrm{~s}, 3 \mathrm{H}, \mathrm{C}\left(\mathrm{CH}_{3}\right)\right), 0.99-$ 1.06 (many lines, $\left.12 \mathrm{H}, \mathrm{C}\left(\mathrm{CH}_{3}\right)+\mathrm{N}\left(\mathrm{CH}_{2} \mathrm{CH}_{3}\right)_{2}\right)+$ $\left.=\mathrm{C}\left(\mathrm{CH}_{2} \mathrm{CH}_{3}\right)\right), \quad 1.89 \quad\left(d,{ }^{4} J(\mathrm{P}-\mathrm{H})=1.5 \mathrm{~Hz}, \quad 3 \mathrm{H}\right.$, $\mathrm{C}\left(\mathrm{CH}_{2} \mathrm{CH}_{3}\right), 2.56\left(q^{3} J(\mathrm{H}-\mathrm{H})=6.6 \mathrm{~Hz}, 2 \mathrm{H}, \mathrm{CH}_{2} \mathrm{CH}_{3}\right)$, $3.10\left(q,{ }^{3} J(\mathrm{H}-\mathrm{H})=6.9 \mathrm{~Hz}, 2 \mathrm{H}, \mathrm{N}\left(\mathrm{CH}_{2} \mathrm{CH}_{3}\right)_{2}\right), 3.55-$ $3.63\left(m, 3 \mathrm{H}, \quad \mathrm{OCH}_{2}+\mathrm{PC} H\right), \quad 4.06\left(t,{ }^{3} \mathrm{~J}(\mathrm{P}-\mathrm{H})=\right.$ $\left.10.5 \mathrm{~Hz}, \quad 2 \mathrm{H}, \quad \mathrm{OCH}_{2}\right) .{ }^{13} \mathrm{C} \quad \mathrm{NMR}:{ }^{\delta} 12.3 \quad(s$, $\left.\mathrm{C}\left(\mathrm{NCH}_{2} \mathrm{CH}_{3}\right)_{2} \mathrm{Et}\right), 13 \cdot 6\left(s,\left(\mathrm{CH}_{2} \mathrm{CH}_{3}\right), 19 \cdot 6,21 \cdot 0(2 s\right.$, $\left.\mathrm{C}\left(\mathrm{CH}_{3}\right)_{2}\right), 23.2\left(d,{ }^{3} \mathrm{~J}(\mathrm{P}-\mathrm{C})=4.4 \mathrm{~Hz}, \mathrm{C}\left(\mathrm{CH}_{2} \mathrm{CH}_{3}\right), 31.9\right.$ $\left(d,{ }^{3} J(\mathrm{P}-\mathrm{C})=5 \cdot 2 \mathrm{~Hz}, C \mathrm{Me}_{2}\right), 43.0\left(s, \mathrm{C}\left(\mathrm{NCH}_{2} \mathrm{CH}_{3}\right)_{2}\right.$ Et), $69.5\left(d,{ }^{1} J(\mathrm{P}-\mathrm{C})=217.9 \mathrm{~Hz}, \mathrm{P} C\right), 74.2$ and 74.3 $\left(2 s, \mathrm{OCH}_{2}\right), 165 \cdot 3\left(d,{ }^{2} J(\mathrm{P}-\mathrm{C})=22 \cdot 1 \mathrm{~Hz}, \mathrm{PCH}=C\right)$. ${ }^{31} \mathrm{P}$ NMR: $\delta 23 \cdot 8$

\subsection{Synthesis of $\beta$-ketophosphonates $\left(\mathrm{OCH}_{2} \mathrm{CMe}_{2} \mathrm{CH}_{2} \mathrm{O}\right) \mathrm{P}(\mathrm{O}) \mathrm{CR}^{3} \mathrm{C}(\mathrm{O}) \mathrm{CHR}^{1} \mathrm{R}^{2}$ (22-26)}

To a solution of allenyl phosphonates 12-16 $(10.0 \mathrm{mmol})$ in dry acetonitrile $(20 \mathrm{~mL})$ was added 
diethylamine $(0.73 \mathrm{~g}, 1.03 \mathrm{~mL}, 10.0 \mathrm{mmol})$ and the mixture stirred for $4 \mathrm{~h}$. The reaction mixture was then treated with $2 \mathrm{~N} \mathrm{HCl}$, stirred for $8 \mathrm{~h}$ and extracted with dichloromethane $\left(\mathrm{CH}_{2} \mathrm{Cl}_{2}\right)(3 \times 30 \mathrm{~mL})$. The $\mathrm{CH}_{2} \mathrm{Cl}_{2}$ layer was dried $\left(\mathrm{Na}_{2} \mathrm{SO}_{4}\right)$, solvent was removed and the residue purified by column chromatography (silica gel; hexane-ethyl acetate) to obtain 22-26.

2.6a $\left(\mathrm{OCH}_{2} \mathrm{CMe}_{2} \mathrm{CH}_{2} \mathrm{O}\right) \mathrm{P}(\mathrm{O}) \mathrm{CH}_{2} \mathrm{C}(\mathrm{O}) \mathrm{Me}$ (22): Yield: $1.94 \mathrm{~g}$ (94\%). M.p.: $85-86^{\circ} \mathrm{C}$. IR (KBr): $1714,1273,1061,1009 \mathrm{~cm}^{-1} .{ }^{1} \mathrm{H}$ NMR: $\delta 0.90$ and $0.99\left(2 s, 6 \mathrm{H}, \mathrm{C}\left(\mathrm{CH}_{3}\right)_{2}\right), 2.30\left(s, 3 \mathrm{H}, \mathrm{C}(\mathrm{O}) \mathrm{CH}_{3}\right), 3.09$ $\left(d,{ }^{2} J(\mathrm{P}-\mathrm{H})=21.5 \mathrm{~Hz}, 2 \mathrm{H}, \mathrm{PCH}_{2}\right), 3.80-4.05(\mathrm{~m}$, $\left.4 \mathrm{H}, \mathrm{OCH}_{2}\right) .{ }^{13} \mathrm{C}$ NMR: $\delta 21.0$ and $21.5\left(2 \mathrm{~s}, \mathrm{C}\left(\mathrm{CH}_{3}\right)_{2}\right)$, $31.5\left(d,{ }^{3} J(\mathrm{P}-\mathrm{C})=4.1 \mathrm{~Hz}, \mathrm{C}(\mathrm{O}) \mathrm{CH}_{3}\right), 32.5\left(d,{ }^{3} \mathrm{~J}(\mathrm{P}-\right.$ $\left.\mathrm{C})=5.3 \mathrm{~Hz}, C \mathrm{Me}_{2}\right), 41.7\left(d,{ }^{1} J(\mathrm{P}-\mathrm{C})=123.9 \mathrm{~Hz}, \mathrm{PC}\right)$, $76 \cdot 1,76 \cdot 2\left(2 s, \mathrm{OCH}_{2}\right), 199 \cdot 3\left(d,{ }^{2} J(\mathrm{P}-\mathrm{C})=6 \cdot 0 \mathrm{~Hz}\right.$, $\left.\mathrm{PCH}_{2} \mathrm{C}(\mathrm{O})\right)$. ${ }^{31} \mathrm{P}$ NMR: $\delta$ 13.8. Anal. Calcd for $\mathrm{C}_{8} \mathrm{H}_{15} \mathrm{O}_{4} \mathrm{P}: \mathrm{C}, 46.60 ; \mathrm{H}, 7.33$. Found: C, 46.84; H, 7.32.

$2.6 \mathrm{~b} \quad\left(\mathrm{OCH}_{2} \mathrm{CMe}_{2} \mathrm{CH}_{2} \mathrm{O}\right) \mathrm{P}(\mathrm{O}) \mathrm{CH}_{2} \mathrm{C}(\mathrm{O}) \mathrm{Et}$

(23):

Yield: $1.87 \mathrm{~g}(85 \%)$. M.p.: $98-100^{\circ} \mathrm{C}$. IR (KBr): $1715,1267,1063,1007 \mathrm{~cm}^{-1} .{ }^{1} \mathrm{H}$ NMR: $\delta 0.93-1.09$ (3 lines, $\left.9 \mathrm{H}, \mathrm{C}\left(\mathrm{CH}_{3}\right)_{2}+\mathrm{CH}_{2} \mathrm{CH}_{3}\right), 2.58\left(q r t,{ }^{3} \mathrm{~J}(\mathrm{H}-\right.$ $\left.\mathrm{H}) \sim 6.0 \mathrm{~Hz}, \quad 2 \mathrm{H}, \quad \mathrm{CH}_{2} \mathrm{CH}_{3}\right), \quad 3.12 \quad\left(d, \quad{ }^{2} \mathrm{~J}(\mathrm{P}-\right.$ $\left.\mathrm{H})=21 \cdot 0 \mathrm{~Hz}, 2 \mathrm{H}, \mathrm{PCH}_{2}\right), 3 \cdot 83-4 \cdot 12\left(m, 4 \mathrm{H}, \mathrm{OCH}_{2}\right)$.

${ }^{13} \mathrm{C}$ NMR: $\delta 7.4\left(s, \mathrm{CH}_{2} \mathrm{CH}_{3}\right), 21.1$ and $21.5(2 s$, $\left.\mathrm{C}\left(\mathrm{CH}_{3}\right)_{2}\right), 32.5\left(d,{ }^{3} J(\mathrm{P}-\mathrm{C})=6.6 \mathrm{~Hz}, C \mathrm{Me}_{2}\right), 37.5(s$, $\left.\mathrm{CH}_{2} \mathrm{CH}_{3}\right), 40.2\left(d,{ }^{1} J(\mathrm{P}-\mathrm{C})=122.6 \mathrm{~Hz}, \mathrm{PC}\right), 76.0$ and $76.1\left(2 s, \mathrm{OCH}_{2}\right), 201.9\left(d,{ }^{2} J(\mathrm{P}-\mathrm{C})=6.1 \mathrm{~Hz}\right.$, $\left.\mathrm{PCH}_{2} \mathrm{C}(\mathrm{O})\right)$. ${ }^{31} \mathrm{P}$ NMR: $\delta$ 14.0. Anal. Calcd for $\mathrm{C}_{9} \mathrm{H}_{17} \mathrm{O}_{4} \mathrm{P}: \mathrm{C}, 49.09 ; \mathrm{H}, 7.78$. Found: $\mathrm{C}, 48.88 ; \mathrm{H}$, 7.75.

2.6c $\left(\mathrm{OCH}_{2} \mathrm{CMe}_{2} \mathrm{CH}_{2} \mathrm{O}\right) \mathrm{P}(\mathrm{O}) \mathrm{CH}_{2} \mathrm{C}(\mathrm{O}) \mathrm{CHMe}_{2}$ (24): Yield: $1.42 \mathrm{~g}(61 \%)$. M.p.: $102-104^{\circ} \mathrm{C}$. IR (KBr): $1713,1474,1267,1059,1009 \mathrm{~cm}^{-1} .{ }^{1} \mathrm{H}$ NMR: $\delta 1 \cdot 00$, $1.10\left(2 s, 6 \mathrm{H}, \mathrm{C}\left(\mathrm{CH}_{3}\right)_{2}\right), 1.06\left(d,{ }^{3} \mathrm{~J}(\mathrm{H}-\mathrm{H})=6.8 \mathrm{~Hz}\right.$, $\left.6 \mathrm{H}, \mathrm{CH}\left(\mathrm{CH}_{3}\right)_{2}\right), 2 \cdot 77-2 \cdot 87\left(q n t,{ }^{3} \mathrm{~J}(\mathrm{H}-\mathrm{H})=6 \cdot 8 \mathrm{~Hz}\right.$, $\left.1 \mathrm{H}, \mathrm{CH}\left(\mathrm{CH}_{3}\right)_{2}\right), 3 \cdot 12\left(d d,{ }^{2} J(\mathrm{P}-\mathrm{H})=19.6 \mathrm{~Hz},{ }^{2} J(\mathrm{H}-\right.$ $\left.\mathrm{H})=2 \cdot 0 \mathrm{~Hz}, 2 \mathrm{H}, \mathrm{PCH}_{2}\right), 3 \cdot 88-4 \cdot 14\left(m, 4 \mathrm{H}, \mathrm{OCH}_{2}\right)$. ${ }^{13} \mathrm{C}$ NMR: $\delta 17.9\left(s, \mathrm{CH}\left(\mathrm{CH}_{3}\right)_{2}\right), 21.2$ and $21.6(2 s$, $\mathrm{C}\left(\mathrm{CH}_{3}\right)_{2}, 32.5\left(d,{ }^{3} \mathrm{~J}(\mathrm{P}-\mathrm{C})=6.6 \mathrm{~Hz}, C \mathrm{Me}_{2}\right), 37.3(s$, $\left.\mathrm{CH}\left(\mathrm{CH}_{3}\right)_{2}\right), 40.9\left(d,{ }^{1} J(\mathrm{P}-\mathrm{C})=104.3 \mathrm{~Hz}, \mathrm{PC}\right), 76 \cdot 0$ and $76.1\left(2 s, \mathrm{OCH}_{2}\right), 205.5\left(d,{ }^{2} J(\mathrm{P}-\mathrm{C})=6.0 \mathrm{~Hz}\right.$, $\left.\mathrm{PCH}_{2} C(\mathrm{O})\right) .{ }^{31} \mathrm{P}$ NMR: $\delta 14 \cdot 2$.

2.6d $\left(\mathrm{OCH}_{2} \mathrm{CMe}_{2} \mathrm{CH}_{2} \mathrm{O}\right) \mathrm{P}(\mathrm{O}) \mathrm{CH}_{2} \mathrm{C}(\mathrm{O}) \mathrm{CH}(\mathrm{Me}) \mathrm{Et}$ (25): Yield: $1.30 \mathrm{~g}(56 \%)$. M.p.: $92-94^{\circ} \mathrm{C}$. IR ( $\left.\mathrm{KBr}\right)$ : $1711,1634,1273,1063,1009 \mathrm{~cm}^{-1} .{ }^{1} \mathrm{H}$ NMR: $\delta 0.81$ $\left(t,{ }^{3} J(\mathrm{H}-\mathrm{H})=7.4 \mathrm{~Hz}, 3 \mathrm{H}, \mathrm{CH}_{2} \mathrm{CH}_{3}\right), 1.00$ and 1.06 $\left(2 s, 6 \mathrm{H}, \mathrm{C}\left(\mathrm{CH}_{3}\right)_{2}\right), 1.07\left(d,{ }^{3} J(\mathrm{H}-\mathrm{H})=6.7 \mathrm{~Hz}, 3 \mathrm{H}\right.$, $\left.\mathrm{CHCH}_{3}\right), 1.27-1.42,1.54-1.73(2 m, 2 \mathrm{H}, \mathrm{CH}(\mathrm{Me})$ $\left.\mathrm{CH}_{2} \mathrm{CH}_{3}\right), 2.69\left(m, 1 \mathrm{H}, \mathrm{CH}\left(\mathrm{CH}_{3}\right) \mathrm{CH}_{2} \mathrm{CH}_{3}\right), 3.20(d$, $\left.{ }^{2} J(\mathrm{P}-\mathrm{H})=22.3 \mathrm{~Hz}, 2 \mathrm{H}, \mathrm{PCH}_{2}\right), 3.89-4.14(\mathrm{~m}, 2 \mathrm{H}$, $\left.\mathrm{OCH}_{2}\right) .{ }^{13} \mathrm{C}$ NMR: $\delta 11.3\left(\mathrm{~s}, \mathrm{CHCH}_{2} \mathrm{CH}_{3}\right), 15 \cdot 2(\mathrm{~s}$, $\left.\mathrm{CH}\left(\mathrm{CH}_{3}\right) \mathrm{CH}_{2} \mathrm{CH}_{3}\right), 21.1$ and $21.6\left(2 s, \mathrm{CMe}_{2}\right), 25.4$ $\left(s, \quad \mathrm{CH}\left(\mathrm{CH}_{3}\right) \mathrm{CH}_{2} \mathrm{CH}_{3}\right), \quad 32.5 \quad\left(d,{ }^{3} J(\mathrm{PC})=7.3 \mathrm{~Hz}\right.$, $\left.C\left(\mathrm{CH}_{3}\right)_{2}\right), 38 \cdot 1\left(d,{ }^{1} J(\mathrm{P}-\mathrm{C})=126 \cdot 1 \mathrm{~Hz}, \mathrm{PC}\right), 48.8(s$, $\left.\mathrm{CH}\left(\mathrm{CH}_{3}\right) \mathrm{CH}_{2} \mathrm{CH}_{3}\right), 76 \cdot 0$ and 76.1 $\left(2 s, \mathrm{OCH}_{2}\right), 205 \cdot 4$ $\left(d,{ }^{2} J(\mathrm{P}-\mathrm{C})=6.0 \mathrm{~Hz}, \mathrm{PCH}_{2} C(\mathrm{O})\right) .{ }^{31} \mathrm{P}$ NMR: $\delta 14.6$.

\section{6e $\left(\mathrm{OCH}_{2} \mathrm{CMe}_{2} \mathrm{CH}_{2} \mathrm{O}\right) \mathrm{P}(\mathrm{O}) \mathrm{CHPhC}(\mathrm{O}) \mathrm{CH}_{2} \mathrm{CH}_{2}$} $\mathrm{CH}_{3}$ (26): Yield: $1.33 \mathrm{~g}(43 \%)$. M.p.: $138-140^{\circ} \mathrm{C}$. IR $(\mathrm{KBr}): 1717,1263,1059,1005 \mathrm{~cm}^{-1} .{ }^{1} \mathrm{H}$ NMR: $\delta$ 0.82 and $1.01\left(2 s, 6 \mathrm{H}, \mathrm{C}\left(\mathrm{CH}_{3}\right)_{2}\right), 0.84\left(t,{ }^{3} J(\mathrm{H}-\mathrm{H})=\right.$ $\left.6.3 \mathrm{~Hz}, 3 \mathrm{H}, \mathrm{CH}_{2} \mathrm{CH}_{2} \mathrm{CH}_{3}\right), 1.58\left(m, 2 \mathrm{H}, \mathrm{CH}_{2} \mathrm{CH}_{2} \mathrm{CH}_{3}\right)$, 2.51-2.64 (m, 2H, $\left.\mathrm{CH}_{2} \mathrm{CH}_{2} \mathrm{CH}_{3}\right), 3.67-3.88(m, 2 \mathrm{H}$, $\left.\mathrm{OCH}_{2}\right), 4.03\left(t,{ }^{3} J(\mathrm{P}-\mathrm{H}) \sim 6.0 \mathrm{~Hz}, 2 \mathrm{H}, \mathrm{OCH}_{2}\right), 4.53$ $\left(d,{ }^{2} J(\mathrm{P}-\mathrm{H})=21.2 \mathrm{~Hz}, 2 \mathrm{H}, \mathrm{PCH}_{2}\right), 7.23-7.49(m, 5 \mathrm{H}$, Ar- $H) .{ }^{13} \mathrm{C}$ NMR: $\delta 13.3\left(s, \mathrm{CH}_{2} \mathrm{CH}_{2} \mathrm{CH}_{3}\right), 16.9(s$, $\left.\mathrm{CH}_{2} \mathrm{CH}_{2} \mathrm{CH}_{3}\right), 21.1$ and $21.7\left(2 \mathrm{~s}, \mathrm{C}\left(\mathrm{CH}_{3}\right)_{2}\right), 32.6(d$, $\left.{ }^{3} J(\mathrm{P}-\mathrm{C})=7 \cdot 4 \mathrm{~Hz}, C \mathrm{Me}_{2}\right), \quad 45 \cdot 2 \quad\left(s, \mathrm{CH}_{2} \mathrm{CH}_{2} \mathrm{CH}_{3}\right)$, $58.5\left(d,{ }^{1} J(\mathrm{P}-\mathrm{C})=129.0 \mathrm{~Hz}, \mathrm{PC}\right), 76.0$ and $76.1(2 d$, ${ }^{3} J(\mathrm{PC})=6 \cdot 7 \mathrm{~Hz}$ each, $\left.2 \mathrm{OCH}_{2}\right), 128 \cdot 1,128 \cdot 8,129 \cdot 6$, $129.9,130.5,202.9\left(d,{ }^{2} J(\mathrm{PC})=4.9 \mathrm{~Hz}, \mathrm{PCH}_{2} C(\mathrm{O})\right)$. ${ }^{31}$ P NMR: $\delta 12 \cdot 3$.

\subsection{HWE reaction using the $\beta$-ketophosphonates 22-23: Synthesis of $\alpha, \beta$-unsaturated ketones $27 a-e$ and $28 a-f$}

To the $\beta$-ketophosphonate 22 or $23(10.0 \mathrm{mmol})$ in dry THF $(20 \mathrm{~mL}), \mathrm{K}_{2} \mathrm{CO}_{3}(1.66 \mathrm{~g}, 12.0 \mathrm{mmol})$ was added and the reaction mixture stirred for $30 \mathrm{~min}$ at room temperature. Then aldehyde $(10.0 \mathrm{mmol})$ was added in one lot and the reaction mixture was refluxed for $24 \mathrm{~h}$. After cooling to room temperature, the reaction mixture was quenched with cold water $(20 \mathrm{~mL})$ and extracted with ether $(2 \times 30 \mathrm{~mL})$. The ether layer was dried $\left(\mathrm{Na}_{2} \mathrm{SO}_{4}\right)$, the solvent removed and the residue was purified by column chromatography (silica gel; hexane-ethyl acetate) to obtain $27 \mathbf{a}-\mathbf{e}$ or $28 \mathbf{a}-\mathbf{f}$.

2.7a $C_{6} H_{4}-4-M e(H) C=C(H) C(O) M e$ (27a): Yield: 1.26 g (79\%). M.p.: liquid. IR (KBr): 1667, 1611 , $1258,1208,1179,980 \mathrm{~cm}^{-1}$. ${ }^{1} \mathrm{H}$ NMR: $\delta 2.36(s, 6 \mathrm{H}$, $\left.\mathrm{Ar}-\mathrm{CH}_{3}+\mathrm{C}(\mathrm{O}) \mathrm{CH}_{3}\right), 6.67\left(d,{ }^{3} \mathrm{~J}(\mathrm{H}-\mathrm{H})=16.6 \mathrm{~Hz}, 1 \mathrm{H}\right.$, $\mathrm{HC}=\mathrm{CHC}(\mathrm{O}) \mathrm{Me}), 7.19\left(d,{ }^{3} J(\mathrm{H}-\mathrm{H})=8.8 \mathrm{~Hz}, 2 \mathrm{H}\right.$, $\mathrm{Ar}-\mathrm{H}$ ), 7.41-7.53 (3 lines, $3 \mathrm{H}, \mathrm{Ar}-\mathrm{H}+\mathrm{C}_{6} \mathrm{H}_{4}-4-\mathrm{Me}-$ 
$H \mathrm{C}=\mathrm{CH}) .{ }^{13} \mathrm{C} \mathrm{NMR}: \delta 21.4\left(s, \mathrm{ArCH}_{3}\right), 27.4(s$, $\left.\mathrm{C}(\mathrm{O}) \mathrm{CH}_{3}\right), 126 \cdot 3,128 \cdot 3,129 \cdot 7,131 \cdot 7,141 \cdot 0,143 \cdot 4$, $198.3(s, C(\mathrm{O}) \mathrm{Me})$. Anal. Calcd for $\mathrm{C}_{11} \mathrm{H}_{12} \mathrm{O}$ : C, $82 \cdot 50 ; \mathrm{H}, 7 \cdot 50$. Found: C, 82.68; H, 7.38.

2.7b $\quad \mathrm{C}_{6} \mathrm{H}_{4}-3-\mathrm{Me}(H) \mathrm{C}=\mathrm{C}(H) \mathrm{C}(\mathrm{O}) \mathrm{Me}$ (27b): Yield: $1.3 \mathrm{~g}$ (81\%). M.p.: liquid. IR (KBr): 1669, 1611 , 1258, $1229,978 \mathrm{~cm}^{-1} .{ }^{1} \mathrm{H}$ NMR: $\delta 2.37(s, 6 \mathrm{H}, \mathrm{Ar}-$ $\left.\mathrm{CH}_{3}+\mathrm{C}(\mathrm{O}) \mathrm{CH}_{3}\right), 6.69\left(d,{ }^{3} \mathrm{~J}(\mathrm{H}-\mathrm{H})=15.6 \mathrm{~Hz}, 1 \mathrm{H}\right.$, $\left.\mathrm{C}_{6} \mathrm{H}_{4}-3-\mathrm{Me}(\mathrm{H}) \mathrm{C}=\mathrm{C} H\right), \quad 7.22-7.35 \quad\left(m, 4 \mathrm{H}, \mathrm{C}_{6} \mathrm{H}_{4}-3-\right.$ $\mathrm{Me}(H) \mathrm{C}=\mathrm{CH}), \quad 7.48 \quad\left(d,{ }^{3} J(\mathrm{H}-\mathrm{H})=15.6 \mathrm{~Hz}, \quad 1 \mathrm{H}\right.$, $\left.\mathrm{C}_{6} \mathrm{H}_{4}-3-\mathrm{Me}(H) \mathrm{C}=\mathrm{CH}\right) .{ }^{13} \mathrm{C}$ NMR: $\delta 21.3 \quad(s, \mathrm{Ar}-$ $\left.\mathrm{CH}_{3}\right), 27 \cdot 5\left(\mathrm{~s}, \mathrm{C}(\mathrm{O}) \mathrm{CH}_{3}\right), 125 \cdot 5,127 \cdot 0,128 \cdot 9,131 \cdot 3$, $134 \cdot 4,138 \cdot 6,143 \cdot 6,198 \cdot 3(s, C(\mathrm{O}) \mathrm{Me})$.

2.7c $\quad \mathrm{C}_{6} \mathrm{H}_{4}-4-\mathrm{OMe}(H) \mathrm{C}=\mathrm{C}(\mathrm{H}) \mathrm{C}(\mathrm{O}) \mathrm{Me}$ (27c): Yield: $1.53 \mathrm{~g}(87 \%)$. M.p.: $62-64^{\circ} \mathrm{C}$. IR (KBr): 1771, 1744 , $1229,1103,959 \mathrm{~cm}^{-1} .{ }^{1} \mathrm{H}$ NMR: $\delta 2.35(s, 3 \mathrm{H}$, $\left.\mathrm{C}(\mathrm{O}) \mathrm{CH}_{3}\right), \quad 3.83 \quad\left(s, 3 \mathrm{H}, \quad \mathrm{OCH}_{3}\right), 6.59 \quad\left(d,{ }^{3} \mathrm{~J}(\mathrm{H}-\right.$ $\left.\mathrm{H})=16 \cdot 3 \mathrm{~Hz}, 1 \mathrm{H}, \mathrm{C}_{6} \mathrm{H}_{4}-4-\mathrm{OMe}(\mathrm{H}) \mathrm{C}=\mathrm{CH}\right), 6.90(d$, ${ }^{3} J(\mathrm{H}-\mathrm{H})=8.7 \mathrm{~Hz}, 2 \mathrm{H}$, Ar-ortho- $\left.H\right), 7.46\left(d,{ }^{3} J(\mathrm{H}-\right.$ $\left.\mathrm{H})=16.3 \mathrm{~Hz}, 1 \mathrm{H}, \mathrm{C}_{6} \mathrm{H}_{4}-4-\mathrm{OMe} H \mathrm{C}=\mathrm{CH}\right), 7.48(d$, $\left.{ }^{3} J(\mathrm{H}-\mathrm{H})=8.7 \mathrm{~Hz}, 2 \mathrm{H}, \mathrm{Ar}-H\right) \cdot{ }^{13} \mathrm{C}$ NMR: $\delta 27.4(s$, $\left.\mathrm{C}(\mathrm{O}) \mathrm{CH}_{3}\right), 55.4\left(s, \mathrm{Ar}-\mathrm{O} C \mathrm{H}_{3}\right), 114.5,125 \cdot 1,127 \cdot 1$, $130.0,143.2,161.6,198.3(s, C(\mathrm{O}) \mathrm{Me}))$; Anal. Calcd for $\mathrm{C}_{11} \mathrm{H}_{12} \mathrm{O}_{2}$ : C, 75.00; H, 6.82. Found: C, 75.22; H, 6.95.

\section{$2.7 \mathrm{~d} \quad 3,4-\mathrm{Cl}_{2}-\mathrm{C}_{6} \mathrm{H}_{3}-(\mathrm{H}) \mathrm{C}=\mathrm{C}(\mathrm{H}) \mathrm{C}(\mathrm{O}) \mathrm{Me}$}

$(27 d):$

Yield: $1.68 \mathrm{~g}(78 \%)$. M.p.: $52-54{ }^{\circ} \mathrm{C}$. IR (KBr): $1672,1615,1470,1258,1134,1030,978 \mathrm{~cm}^{-1} .{ }^{1} \mathrm{H}$ NMR: $\delta 2.32\left(s, 3 \mathrm{H}, \mathrm{C}(\mathrm{O}) \mathrm{CH}_{3}\right), 6.62\left(d,{ }^{3} J(\mathrm{H}-\right.$ $\left.\mathrm{H})=16.6 \mathrm{~Hz}, \quad 1 \mathrm{H}, \quad \mathrm{C}_{6} \mathrm{H}_{3}-3,4-\mathrm{Cl}_{2}(\mathrm{H}) \mathrm{C}=\mathrm{CH}\right), \quad 7.29-$ $7.54\left(m, 4 \mathrm{H}, \quad 3 \mathrm{Ar}-\mathrm{H}+\mathrm{C}_{6} \mathrm{H}_{3}-3,4-\mathrm{Cl}_{2} \mathrm{HC}=\mathrm{CH}\right) .{ }^{13} \mathrm{C}$ NMR: $\delta 27.8\left(s, \mathrm{C}(\mathrm{O}) C \mathrm{H}_{3}\right), 127 \cdot 1,128.4,129 \cdot 7$, $130 \cdot 9,133 \cdot 2,134 \cdot 2,134 \cdot 6,140 \cdot 2,197 \cdot 4(s, C(\mathrm{O}) \mathrm{Me})$.

2.7e $\quad C_{14} H_{9}(H) C=C(H) C(O) M e(27 e):$ Yield: $1.92 \mathrm{~g}$ (78\%). M.p.: $100-102^{\circ} \mathrm{C}$. IR (KBr): 1660,1653 , $1541,1520,1362,1250,988 \mathrm{~cm}^{-1} .{ }^{1} \mathrm{H}$ NMR: $\delta 2 \cdot 54$ $\left(s, 3 \mathrm{H}, \mathrm{C}(\mathrm{O}) \mathrm{CH}_{3}\right), 6.68\left(d,{ }^{3} \mathrm{~J}(\mathrm{H}-\mathrm{H})=16.6 \mathrm{~Hz}, 1 \mathrm{H}\right.$, $\left.\mathrm{C}_{14} \mathrm{H}_{9}(\mathrm{H}) \mathrm{C}=\mathrm{C} H\right), 7.46-7.50(m, 4 \mathrm{H}, \operatorname{Ar}-H), 7.94-$ $7.80(m, 2 \mathrm{H}, \mathrm{Ar}-H), 8 \cdot 15-8.20(m, 2 \mathrm{H}, \mathrm{Ar}-H), 8.38$ $(s, 1 \mathrm{H}, \quad \operatorname{Ar}-H), 8.42\left(d,{ }^{3} J(\mathrm{H}-\mathrm{H})=16.6 \mathrm{~Hz}, 1 \mathrm{H}\right.$, $\left.\mathrm{C}_{14} \mathrm{H}_{9}(H) \mathrm{C}=\mathrm{CH}\right) .{ }^{13} \mathrm{C}$ NMR: $\delta 27.9 \quad\left(s, \mathrm{C}(\mathrm{O}) \mathrm{CH}_{3}\right)$, $125 \cdot 1,125 \cdot 4,126 \cdot 4,128 \cdot 5,128 \cdot 9,129 \cdot 2,129 \cdot 4$, $131 \cdot 3,135 \cdot 8,140 \cdot 3,197 \cdot 7(s, C(\mathrm{O}) \mathrm{Me})$.

2.7f $\quad\left(4-\mathrm{Me}-\mathrm{C}_{6} \mathrm{H}_{4}\right)(H) \mathrm{C}=\mathrm{C}(\mathrm{H}) \mathrm{C}(\mathrm{O}) \mathrm{Et}$ (28a): Yield: 1.27 g (73\%). M.p.: Liquid. IR (KBr): 1661, 1611 , 1362, $1192,1119,988 \mathrm{~cm}^{-1}$. ${ }^{1} \mathrm{H}$ NMR: $\delta 1 \cdot 16(t$, $\left.{ }^{3} J(\mathrm{H}-\mathrm{H})=7.4 \mathrm{~Hz}, 3 \mathrm{H}, \mathrm{CH}_{2} \mathrm{CH}_{3}\right), 2.36(s, 3 \mathrm{H}, \mathrm{Ar}-$ $\left.\mathrm{CH}_{3}\right), 2.67\left(q,{ }^{3} \mathrm{~J}(\mathrm{H}-\mathrm{H})=7.4 \mathrm{~Hz}, 2 \mathrm{H}, \mathrm{CH}_{2} \mathrm{CH}_{3}\right), 6.69$ $\left(d,{ }^{3} \mathrm{~J}(\mathrm{H}-\mathrm{H})=16 \cdot 0 \mathrm{~Hz}, 1 \mathrm{H}, \mathrm{HC}=\mathrm{C} H \mathrm{C}(\mathrm{O}) \mathrm{Et}\right), 7 \cdot 18$ $\left(d,{ }^{3} J(\mathrm{H}-\mathrm{H})=7.8 \mathrm{~Hz}, 2 \mathrm{H}\right.$, Ar-ortho- $\left.H\right), 7.42(d$, ${ }^{3} J(\mathrm{H}-\mathrm{H})=7.8 \mathrm{~Hz}, 2 \mathrm{H}$, Ar-meta- $\left.H\right), 7.53(d, 3 \mathrm{H}$, $\left.{ }^{3} \mathrm{~J}(\mathrm{H}-\mathrm{H})=16.0 \mathrm{~Hz}, 1 \mathrm{H}, \quad \mathrm{C}_{6} \mathrm{H}_{4}-4-\mathrm{Me}(H) \mathrm{C}=\mathrm{CH}\right) .{ }^{13} \mathrm{C}$ NMR: $\delta 8.2\left(s, \mathrm{CH}_{2} \mathrm{CH}_{3}\right), 21.4\left(s, \mathrm{Ar}-\mathrm{CH}_{3}\right), 33.9(s$, $\left.\mathrm{CH}_{2} \mathrm{CH}_{3}\right), 125 \cdot 2,128 \cdot 3,129 \cdot 7,131 \cdot 9,140 \cdot 8,142 \cdot 2$, $200 \cdot 9(s, C(\mathrm{O}) \mathrm{Et})$.

$2.7 \mathrm{~g} \quad\left(\mathrm{C}_{6} \mathrm{H}_{4}-4-\mathrm{OMe}\right)(H) \mathrm{C}=\mathrm{C}(H) \mathrm{C}(\mathrm{O}) \mathrm{Et}(\mathbf{2 8 b}):$ Yield: 1.68 g (89\%). M.p.: $48-50^{\circ} \mathrm{C}$. IR (KBr): 1684,1657 , $1601,1572,1512,1254,1177,1119,1026,988$, $831 \mathrm{~cm}^{-1} .{ }^{1} \mathrm{H}$ NMR: $\delta 1 \cdot 13\left(t,{ }^{3} J(\mathrm{H}-\mathrm{H})=6.8 \mathrm{~Hz}, 3 \mathrm{H}\right.$, $\left.\mathrm{CH}_{2} \mathrm{CH}_{3}\right), 2.64\left(q,{ }^{3} J(\mathrm{H}-\mathrm{H})=6.8 \mathrm{~Hz}, 2 \mathrm{H}, \mathrm{CH}_{2} \mathrm{CH}_{3}\right)$, $3.80\left(s, 3 \mathrm{H}, \mathrm{Ar}-\mathrm{OCH} \mathrm{H}_{3}\right), 6.59\left(d,{ }^{3} J(\mathrm{H}-\mathrm{H})=16.6 \mathrm{~Hz}\right.$, $1 \mathrm{H}, \mathrm{HC}=\mathrm{C}(H) \mathrm{C}(\mathrm{O}) \mathrm{Et}), 6.87\left(d,{ }^{3} J(\mathrm{H}-\mathrm{H})=8.8 \mathrm{~Hz}\right.$, $2 \mathrm{H}$, Ar-Ortho- $H$ ), 7.43-7.53 ( $m, 3 \mathrm{H}, 2 \mathrm{Ar}-\mathrm{H}+\mathrm{C}_{6} \mathrm{H}_{4}-$ $4-\mathrm{OMe}(H) \mathrm{C}=\mathrm{CH}) .{ }^{13} \mathrm{C}$ NMR: $\delta 8.3 \quad\left(s, \mathrm{CH}_{2} \mathrm{CH}_{3}\right)$, $33.9\left(s, \mathrm{CH}_{2} \mathrm{CH}_{3}\right), 55.3\left(s, \mathrm{Ar}-\mathrm{O} C \mathrm{H}_{3}\right), 114.4,123.9$, $127 \cdot 3,129 \cdot 9,141 \cdot 9,161 \cdot 5,200 \cdot 8(s, C(\mathrm{O}) \mathrm{Et})$.

2.7h $\quad C_{6} H_{5} C H=C H-(H) C=C(H) C(O) E t$ (28c): Yield: $1.21 \mathrm{~g}(65 \%)$. M.p.: Liquid. IR (KBr): 1678, 1622, $1589,1451,1358,1287,1192,1123,999,748 \mathrm{~cm}^{-1}$. ${ }^{1} \mathrm{H}$ NMR: $\delta 1.14\left(t,{ }^{3} J(\mathrm{H}-\mathrm{H})=6.7 \mathrm{~Hz}, 3 \mathrm{H}, \mathrm{CH}_{2} \mathrm{CH}_{3}\right)$, $2.61\left(q,{ }^{3} J(\mathrm{H}-\mathrm{H})=6.7 \mathrm{~Hz}, 2 \mathrm{H}, \mathrm{CH}_{2} \mathrm{CH}_{3}\right), 6.27(d$, $\left.{ }^{3} J(\mathrm{H}-\mathrm{H})=16.0 \mathrm{~Hz}, \quad 1 \mathrm{H}, \quad(\mathrm{H}) \mathrm{C}=\mathrm{C} H \mathrm{C}(\mathrm{O}) \mathrm{Et}\right), \quad 6.85-$ $6.90\left(m, 2 \mathrm{H}, \mathrm{C}_{6} \mathrm{H}_{5}(\mathrm{H}) \mathrm{C}=\mathrm{C}(\mathrm{H})-(H) \mathrm{C}=\mathrm{CH}+1 \mathrm{Ar}-H\right)$, 7.31-7.48 $\quad\left(m, \quad 6 \mathrm{H}, \quad \mathrm{C}_{6} \mathrm{H}_{5}(H) \mathrm{C}=\mathrm{C}(H)-(\mathrm{H}) \mathrm{C}=\mathrm{CH}+\right.$ $4 \mathrm{Ar}-\mathrm{H}) .{ }^{13} \mathrm{C}$ NMR: $\delta 8.3\left(s, \mathrm{CH}_{2} \mathrm{CH}_{3}\right), 33.9(s$, $\left.\mathrm{CH}_{2} \mathrm{CH}_{3}\right), 126 \cdot 8,127 \cdot 2,128 \cdot 8,129 \cdot 0,129 \cdot 4,131 \cdot 2$, $136 \cdot 2,141 \cdot 0,142 \cdot 1,152 \cdot 4,193 \cdot 3(s, C(\mathrm{O}) \mathrm{Et})$.

$2.7 \mathrm{i} \quad C_{14} H_{9}(H) C=C(H) C(O) E t(28 d):$ Yield: $1.77 \mathrm{~g}$ (68\%). M.p.: $94-98^{\circ} \mathrm{C}$. IR (KBr): 1661, 1616, 1194, $1019,982 \mathrm{~cm}^{-1} .{ }^{1} \mathrm{H}$ NMR: $\delta 1.27\left(t,{ }^{3} J(\mathrm{H}-\mathrm{H})=7.6 \mathrm{~Hz}\right.$, $\left.3 \mathrm{H}, \quad \mathrm{CH}_{2} \mathrm{CH}_{3}\right), 2.83\left(q,{ }^{3} J(\mathrm{H}-\mathrm{H})=7.6 \mathrm{~Hz}, 2 \mathrm{H}\right.$, $\left.\mathrm{CH}_{2} \mathrm{CH}_{3}\right), 6.71 \quad\left(d,{ }^{3} J(\mathrm{H}-\mathrm{H})=16.4 \mathrm{~Hz}, 1 \mathrm{H}, \mathrm{HC}=\right.$ CHC(O)Et), 7.44-7.50 ( $m, 4 \mathrm{H}, \mathrm{Ar}-H), 7.97-8.02(\mathrm{~m}$, $2 \mathrm{H}, \operatorname{Ar}-H), 8 \cdot 17-8.21(m, 2 \mathrm{H}, \operatorname{Ar}-H), 8.43(s, 1 \mathrm{H}$, $\mathrm{Ar}-H), 8.48\left(d,{ }^{3} J(\mathrm{H}-\mathrm{H})=16.4 \mathrm{~Hz}, 1 \mathrm{H}, \mathrm{C}_{14} \mathrm{H}_{9}(H)\right.$ $\mathrm{C}=\mathrm{CH}) .{ }^{13} \mathrm{C}$ NMR: $\delta 8.2 \quad\left(s, \mathrm{CH}_{2} \mathrm{CH}_{3}\right), 34.6(s$, $\left.\mathrm{CH}_{2} \mathrm{CH}_{3}\right), 125 \cdot 2,125 \cdot 4,126 \cdot 3,128 \cdot 2,128 \cdot 9,129 \cdot 5$, $131 \cdot 4,134 \cdot 9,139 \cdot 2,200 \cdot 8(s, C(\mathrm{O}) \mathrm{Et})$.

\section{7j $\mathrm{C}_{5} \mathrm{H}_{5} \mathrm{FeC}_{5} \mathrm{H}_{4}(\mathrm{H}) \mathrm{C}=\mathrm{C}(\mathrm{H}) \mathrm{C}(\mathrm{O}) \mathrm{CH}_{2} \mathrm{CH}_{3} \quad(28 \mathrm{e})$ :}

Yield: $1.82 \mathrm{~g}(68 \%)$. M.p.: $98-100^{\circ} \mathrm{C}$. IR (KBr): $1686,1657,1605,1360,1125,1034,980 \mathrm{~cm}^{-1} .{ }^{1} \mathrm{H}$ NMR: $\delta 1.14\left(t,{ }^{3} \mathrm{~J}(\mathrm{H}-\mathrm{H})=6.8 \mathrm{~Hz}, 3 \mathrm{H}, \mathrm{CH}_{2} \mathrm{CH}_{3}\right)$, $2.58\left(q,{ }^{3} \mathrm{~J}(\mathrm{H}-\mathrm{H})=6.8 \mathrm{~Hz}, 2 \mathrm{H}, \mathrm{CH}_{2} \mathrm{CH}_{3}\right), 4.13(s$, 
$5 \mathrm{H}$, ferrocenyl- $H), 4.41-4.42(m, 2 \mathrm{H}$, ferrocenyl- $H)$, 4.48-4.49 ( $m, 2 \mathrm{H}$, ferrocenyl- $H), 6.34\left(d,{ }^{3} J(\mathrm{H}-\right.$ $\mathrm{H})=15.6 \mathrm{~Hz}, 1 \mathrm{H}, \mathrm{HC}=\mathrm{CH}(\mathrm{CO}) \mathrm{Et}), 7.44\left(d,{ }^{3} J(\mathrm{H}-\right.$ $\left.\mathrm{H})=15.6 \mathrm{~Hz}, \quad 1 \mathrm{H}, \quad \mathrm{C}_{5} \mathrm{H}_{5} \mathrm{FeC}_{5} \mathrm{H}_{4}(H) \mathrm{C}=\mathrm{CH}\right) . \quad{ }^{13} \mathrm{C}$ NMR: $\delta 8.5\left(s, \mathrm{CH}_{2} \mathrm{CH}_{3}\right), 33.6\left(s, \mathrm{CH}_{2} \mathrm{CH}_{3}\right), 68.7$, $69.7,70.9,78.9$ (ferrocenyl- $C), 123.6(s, \mathrm{HC}=\mathrm{CH}$ (CO)Et), $143.6\left(s, \mathrm{C}_{5} \mathrm{H}_{5} \mathrm{FeC}_{5} \mathrm{H}_{4} \mathrm{H} \mathrm{C}=\mathrm{CH}\right), 200 \cdot 2(s$, $C(\mathrm{O}) \mathrm{Et}$ ). Anal. Calcd. for $\mathrm{C}_{15} \mathrm{H}_{16} \mathrm{FeO}: \mathrm{C}, 67 \cdot 16 ; \mathrm{H}$, 5.97. Found: C, 67.34; H, $6 \cdot 08$.

$2.7 \mathrm{k} \quad E t C(O) C(H)=C(H)-C_{6} H_{4}-(H) C=C(H) C(O) E t$ (28f): Yield: $1.91 \mathrm{~g}$ (79\%). M.p.: $112-116^{\circ} \mathrm{C}$. IR (KBr): 1657, 1366, 1192, 1065, $990 \mathrm{~cm}^{-1} .{ }^{1} \mathrm{H}$ NMR: $\delta 1.16\left(t,{ }^{3} \mathrm{~J}(\mathrm{H}-\mathrm{H})=7.8 \mathrm{~Hz}, 3 \mathrm{H}, \mathrm{CH}_{2} \mathrm{CH}_{3}\right), 2.68(q$, $\left.{ }^{3} J(\mathrm{H}-\mathrm{H})=7.8 \mathrm{~Hz}, 2 \mathrm{H}, \quad \mathrm{CH}_{2} \mathrm{CH}_{3}\right), 6.75\left(d,{ }^{3} J(\mathrm{H}-\right.$ $\mathrm{H})=16.6 \mathrm{~Hz}, 1 \mathrm{H}, \mathrm{HC}=\mathrm{C}(H) \mathrm{C}(\mathrm{O}) \mathrm{Et}), 7.48-7.56(3$ lines, $\left.3 \mathrm{H}, 2 \mathrm{Ar}-H+(\mathrm{H}) \mathrm{C}=\mathrm{C}(H)-\mathrm{C}_{6} \mathrm{H}_{4}-(H) \mathrm{C}=\mathrm{C}(\mathrm{H})\right)$. ${ }^{13} \mathrm{C}$ NMR: $\delta 8 \cdot 1\left(s, \mathrm{CH}_{2} \mathrm{CH}_{3}\right), 34.2\left(s, \mathrm{CH}_{2} \mathrm{CH}_{3}\right)$, $126 \cdot 5,128 \cdot 7,136 \cdot 5,140 \cdot 9,200 \cdot 5(s, C(\mathrm{O}) \mathrm{Et})$. Anal. Calcd. for $\mathrm{C}_{16} \mathrm{H}_{18} \mathrm{O}_{2}$ : C, 79.34; H, 7.44. Found: C, 79.48, H, 7.32.

2.8 Allylation of $\beta$-ketophosphonates 22-23:

Isolation of allylated products $\left(\mathrm{OCH}_{2} \mathrm{CMe}_{2} \mathrm{CH}_{2} \mathrm{O}\right)$ $\mathrm{P}(\mathrm{O}) \mathrm{CH}_{2} \mathrm{C}(\mathrm{OH})\left(\mathrm{CH}_{2} \mathrm{CH}=\mathrm{CH}\right) \mathrm{CH}_{2} \mathrm{R}[\mathrm{R}=\mathrm{H}(29)$, $\mathrm{Me}$ (30)]

To a solution of $\beta$-ketophosphonate $\mathbf{2 2}$ or $\mathbf{2 3}$ $(1.0 \mathrm{mmol})$ in dichloromethane $(20 \mathrm{~mL})$ kept over molecular sieves ( $4 \AA, \sim 0.5 \mathrm{~g}$ ), diallyltin dibromide $(0.33 \mathrm{~g}, 1.0 \mathrm{mmol})^{13}$ was added via syringe at room temperature and the mixture stirred for $20 \mathrm{~h}$. The reaction mixture was quenched with $8 \%$ aq. $\mathrm{NaOH}$ $(20 \mathrm{~mL})$ and extracted with dichloromethane $(2 \times$ $30 \mathrm{~mL})$. The organic layer was dried $\left(\mathrm{Na}_{2} \mathrm{SO}_{4}\right)$ and the solvent was removed to obtain the crude $\beta$ hydroxyphosphonate $\mathbf{2 9}$ or $\mathbf{3 0}$. These were purified by column chromatography (silica gel; hexane-ethyl acetate).

2.8a Compound 29: Yield: 0.12 g (49\%). M.p.: 82-84 ${ }^{\circ} \mathrm{C}$. IR (KBr): $3349,1642,1260,1067 \mathrm{~cm}^{-1}$. ${ }^{1} \mathrm{H}$ NMR: $\delta 1.00,1.11\left(2 s, 6 \mathrm{H}, \mathrm{C}\left(\mathrm{CH}_{3}\right)_{2}\right), 1 \cdot 36(s$, $\left.3 \mathrm{H}, \mathrm{C}(\mathrm{OH}) \mathrm{CH}_{3}\right), 2.08\left(d d,{ }^{2} J(\mathrm{H}-\mathrm{H})=10.9 \mathrm{~Hz},{ }^{2} J(\mathrm{P}-\right.$ $\left.\mathrm{H})=17.3 \mathrm{~Hz}, 2 \mathrm{H}, \mathrm{PCH}_{2}\right), 2.37\left(d,{ }^{3} \mathrm{~J}(\mathrm{H}-\mathrm{H})=7.3 \mathrm{~Hz}\right.$, $\left.2 \mathrm{H}, \quad \mathrm{CH}_{2} \mathrm{CH}=\mathrm{CH}_{2}\right), 3.79\left(d d,{ }^{2} J(\mathrm{H}-\mathrm{H})=12.6 \mathrm{~Hz}\right.$, $\left.{ }^{3} J(\mathrm{P}-\mathrm{H})=11.3 \mathrm{~Hz}, 2 \mathrm{H}, \quad \mathrm{OCH}_{\mathrm{A}} \mathrm{H}_{\mathrm{B}}\right), 4 \cdot 20 \quad\left(t,{ }^{3} J(\mathrm{P}-\right.$ $\left.\mathrm{H})=11.3 \mathrm{~Hz}, 2 \mathrm{H}, \mathrm{OCH}_{\mathrm{A}} H_{\mathrm{B}}\right), 5 \cdot 04\left(d,{ }^{3} J(\mathrm{H}-\mathrm{H})=\right.$ $\left.13.0 \mathrm{~Hz}, 2 \mathrm{H}, \mathrm{CH}_{2} \mathrm{CH}=\mathrm{CH}_{2}\right), 6.82\left(m, 1 \mathrm{H}, \mathrm{CH}_{2} \mathrm{CH}=\right.$ $\left.\mathrm{CH}_{2}\right) .{ }^{13} \mathrm{C}$ NMR: $\delta 21.3,21.7\left(2 s, \mathrm{C}\left(\mathrm{CH}_{3}\right)_{2}\right), 28.2(d$, $\left.{ }^{3} J(\mathrm{PC})=9.4 \mathrm{~Hz}, \mathrm{CH}(\mathrm{OH}) \mathrm{CH}_{3}\right), 32.7\left(s, C \mathrm{Me}_{2}\right), 35.5$ $\left(d,{ }^{1} J(\mathrm{PC})=131.3 \mathrm{~Hz}, \quad \mathrm{P} C\right), \quad 47.8 \quad\left(d,{ }^{3} J(\mathrm{P}-\mathrm{C})=\right.$ $\left.12.3 \mathrm{~Hz}, \mathrm{CH}_{2} \mathrm{CH}=\mathrm{CH}_{2}\right), 70.2\left(d,{ }^{2} J(\mathrm{P}-\mathrm{C})=4.8 \mathrm{~Hz}\right.$, $\mathrm{CH}(\mathrm{OH})), \quad 74.8, \quad 75.0 \quad\left(2 s, \quad \mathrm{OCH}_{2}\right), \quad 118.6 \quad(s$, $\left.\mathrm{CH}_{2} \mathrm{CH}=\mathrm{CH}_{2}\right), 133.8\left(s, \mathrm{CH}_{2} \mathrm{CH}=\mathrm{CH}_{2}\right) .{ }^{31} \mathrm{P}$ NMR: $\delta$ 25.9 .

2.8b Compound 30: Yield: $0.16 \mathrm{~g}$ (62\%). M.p.: 74-76 ${ }^{\circ} \mathrm{C}$. IR (KBr): 3434, 1647, 1240, 1060, $1015 \mathrm{~cm}^{-1}$. ${ }^{1} \mathrm{H}$ NMR: $\delta 0.88\left(t,{ }^{3} J(\mathrm{H}-\mathrm{H})=7.2 \mathrm{~Hz}\right.$, $\left.3 \mathrm{H}, \mathrm{CH}_{2} \mathrm{CH}_{3}\right), 0.97,1.08\left(2 s, 6 \mathrm{H}, \mathrm{C}\left(\mathrm{CH}_{3}\right)_{2}\right), 1.62(q$, $\left.{ }^{3} \mathrm{~J}(\mathrm{H}-\mathrm{H})=7.2 \mathrm{~Hz}, \quad 3 \mathrm{H}, \quad \mathrm{CH}_{2} \mathrm{CH}_{3}\right), 2.05 \quad\left(d,{ }^{2} J(\mathrm{P}-\right.$ $\left.\mathrm{H})=17.7 \mathrm{~Hz}, \quad 2 \mathrm{H}, \quad \mathrm{PC} H_{2}\right), \quad 2.34 \quad\left(d, \quad{ }^{3} J(\mathrm{H}-\mathrm{H})=\right.$ $\left.7.3 \mathrm{~Hz}, 2 \mathrm{H}, \mathrm{CH}_{2} \mathrm{CH}=\mathrm{CH}_{2}\right), 3.76\left(t,{ }^{3} J(\mathrm{P}-\mathrm{H})={ }^{2} J(\mathrm{H}-\right.$ $\left.\mathrm{H})=13.7 \mathrm{~Hz}, \quad 2 \mathrm{H}, \quad \mathrm{OCH}_{\mathrm{A}} \mathrm{H}_{\mathrm{B}}\right), 4 \cdot 16 \quad\left(t,{ }^{3} \mathrm{~J}(\mathrm{P}-\mathrm{H})=\right.$ $\left.10.2 \mathrm{~Hz}, 2 \mathrm{H}, \mathrm{OCH}_{\mathrm{A}} H_{\mathrm{B}}\right), 5.07\left(d,{ }^{3} J(\mathrm{H}-\mathrm{H})=13.3 \mathrm{~Hz}\right.$, $\left.2 \mathrm{H}, \mathrm{CH}_{2} \mathrm{CH}=\mathrm{CH}_{2}\right), 6.78\left(m, 1 \mathrm{H}, \mathrm{CH}_{2} \mathrm{CH}=\mathrm{CH}_{2}\right) .{ }^{13} \mathrm{C}$ NMR: $\delta 7.8\left(s, \mathrm{CH}_{2} \mathrm{CH}_{3}\right), 21 \cdot 3,21.7\left(2 s, \mathrm{C}\left(\mathrm{CH}_{3}\right)_{2}\right)$, $32.4\left(d,{ }^{3} J(\mathrm{P}-\mathrm{C})=5.8 \mathrm{~Hz}, C \mathrm{Me}_{2}\right), 32.9\left(d,{ }^{3} J(\mathrm{P}-\right.$ C) $\left.=9.1 \mathrm{~Hz}, \quad \mathrm{CH}(\mathrm{OH}) \mathrm{CH}_{2} \mathrm{CH}_{3}\right), \quad 33.7 \quad\left(d, \quad{ }^{2} \mathrm{~J}(\mathrm{P}-\right.$ C) $\left.=131.0 \mathrm{~Hz}, \mathrm{PCH}_{2}\right), 44.2\left(d,{ }^{3} J(\mathrm{P}-\mathrm{C})=9.9 \mathrm{~Hz}\right.$, $\left.\mathrm{CH}_{2} \mathrm{CH}=\mathrm{CH}_{2}\right), 72.3\left(d,{ }^{2} J(\mathrm{P}-\mathrm{C})=4.8 \mathrm{~Hz}, \mathrm{CH}(\mathrm{OH})\right)$, $74.8,74.9 \quad\left(2 s, \quad \mathrm{OCH}_{2}\right), \quad 118.4 \quad\left(s, \mathrm{CH}_{2} \mathrm{CH}=\mathrm{CH}_{2}\right)$, $133.7\left(s, \mathrm{CH}_{2} \mathrm{CH}=\mathrm{CH}_{2}\right) .{ }^{31} \mathrm{P}$ NMR: $\delta 26.4$.

\section{$2.9 X$-ray structural analysis of 6, 9, 12, 18 and 24}

X-ray data were collected on a Bruker AXS SMART diffractometer using Mo-K $_{\alpha}(\lambda=0.71073 \AA)$ radiation. The structures were solved by direct methods; ${ }^{14}$ all non-hydrogen atoms were refined anisotropically. The allenic $\mathrm{C}-\mathrm{H}$ hydrogen atoms were located by difference Fourier maps in both the cases, and refined isotropically. In the case of $\mathbf{6}$, the $\mathrm{O}-\mathrm{H}$ hydrogen was also located by difference Fourier map, and refined isotropically. All the other hydrogen atoms were placed geometrically and refined using a riding model with $\mathrm{CH}_{2}$ (methylene) constrained to $0.97 \AA$ with $\mathrm{U}_{\text {iso }}(\mathrm{H})=1 \cdot 2 \mathrm{U}_{\mathrm{eq}}(\mathrm{C})$, and $\mathrm{CH}_{3}$ constrained to $0.96 \AA$ with $\mathrm{U}_{\text {iso }}(\mathrm{H})=1.5 \mathrm{U}_{e q}(\mathrm{C})$. In compound $\mathbf{1 2}$ pseudosymmetry is indicated, but we could not refine the structure in any other space group; the Flack parameter using 'twin' command was reduced to zero. The quality of data for $\mathbf{1 8}$ was not good, but the structure could be unambiguously characterized.

\subsection{Crystal data}

Compound 6: $\mathrm{C}_{9} \mathrm{H}_{15} \mathrm{O}_{4} \mathrm{P}, M=218 \cdot 18$, Monoclinic, space group $P 2{ }_{1} / n, a=8.7632(8), b=9.3504(9)$, $c=13.8022(13) \quad \AA, \quad \beta=105.62(10), \quad V=1089.18$ (18) $\AA^{3}, \quad Z=4, \quad \rho=1.331 \mathrm{~g} \mathrm{~cm}^{-3}, \quad F(000)=464$, 
$\mu=0.240 \mathrm{~mm}^{-1}$, data/restraints/parameters: $2634 / 0 /$ 138. $S$ (all data) $=1.024, R$ indices $(I>2 \sigma(I))$ : $R 1=0 \cdot 0448, w R 2$ (all data) $=0 \cdot 1314$. Max. $/ \mathrm{min}$. residual electron density $\left(\mathrm{e} \AA^{-3}\right) 0 \cdot 381 /-0.251$.

2.10a Compound 9: $\quad \mathrm{C}_{11} \mathrm{H}_{14} \mathrm{O}_{6} \mathrm{P}_{2}, \quad M=304 \cdot 16$, Tetragonal, space group $I 4_{1} / a, a=12.9203(4), b=$ $12.9203(4), c=16 \cdot 8163(11) \AA, \quad V=2807.2(2) \AA^{3}$, $Z=8, \rho=1.439 \mathrm{~g} \mathrm{~cm}^{-3}, F_{000}=1264, \mu=0.328 \mathrm{~mm}^{-1}$, data/restraints/parameters: $1369 / 0 / 115 . S$ (all data $)=$ 1.095. $R$ indices $(I>2 \sigma(I)): R 1=0.0334, w R 2$ (all data $=0.0943$. Max. $/$ min. residual electron density $\left(\mathrm{e} \AA^{-3}\right) 0 \cdot 239 /-0 \cdot 240$.

$2.10 \mathrm{~b}$ Compound 12: $\mathrm{C}_{8} \mathrm{H}_{13} \mathrm{O}_{3} \mathrm{P}, M=188 \cdot 15$, orthorhombic, space group $P 2_{1} 2_{1} 2_{1}, a=8.8325(5)$, $b=8.9436(5), c=12.0211(7) \AA, V=949.60(9) \AA^{3}$, $Z=4, \rho=1.316 \mathrm{~g} \mathrm{~cm}^{-3}, F_{000}=400, \mu=0.256 \mathrm{~mm}^{-1}$, data/restraints/parameters: $1678 / 0 / 118 . S($ all data $)=$ 1.012. $R$ indices $(I>2 \sigma(I)): R 1=0.0334, w R 2$ (all data) $=0.0980$. Flack parameter (using 'twin' command in SHELXL) 0.00. Max./min. residual electron density $\left(\mathrm{e}^{-3}\right) 0 \cdot 189 /-0 \cdot 258$.

2.10c Compound 18: $\mathrm{C}_{20} \mathrm{H}_{34} \mathrm{O}_{6} \mathrm{P}_{2}, M=432.41$, orthorhombic, space group Pbca, $a=19.684(4), b=$ $11.340(2), c=41.629(8) \AA, V=9292(3) \AA^{3}, Z=16$, $\rho=1.236 \mathrm{~g} \mathrm{~cm}^{-3}, F_{000}=3712, \mu=0.218 \mathrm{~mm}^{-1}$, data $/$ restraints/parameters: 8181/0/521. $S$ (all data) $=$ 0.977. $R$ indices $(I>2 \sigma(I)): R 1=0.0961, w R 2$ (all data $=0.2331$. Max. $/$ min. residual electron density $\left(\mathrm{e} \AA^{-3}\right) 0 \cdot 786 /-0 \cdot 286$.

2.10d Compound 24: $\mathrm{C}_{20} \mathrm{H}_{38} \mathrm{O}_{8} \mathrm{P}_{2}, \quad M=468.44$, monoclinic, space group $P 2_{1}, a=6.9687(9), b=$ 19.661(3), $c=9.0871(12) \AA, \quad \beta=96.102(2)^{\circ}, \quad V=$ $1238.0(3) \AA^{3}, Z=2, \rho=1.257 \mathrm{~g} \mathrm{~cm}^{-3}, F_{000}=504$, $\mu=0.215 \mathrm{~mm}^{-1}$, data/restraints/parameters: $4345 / 1 /$ 279. $S$ (all data) $=1 \cdot 225$. Flack parameter (using 'twin' command in SHELXL) $0 \cdot 13(15) . R$ indices $(I>2 \sigma(I)): R 1=0.0811, w R 2$ (all data) $=0 \cdot 1646$. Max. $/$ min. residual electron density $\left(\mathrm{e} \AA^{-3}\right) 0 \cdot 525 /-0 \cdot 257$.

Further details as CIF files are available from the Cambridge Crystallographic Data Centre, 12 Union Road, Cambridge CB2 1EZ, UK on request, quoting the deposition numbers CCDC 695272-695276.

\section{Results and discussion}

The reaction of $\left(\mathrm{OCH}_{2} \mathrm{CMe}_{2} \mathrm{CH}_{2} \mathrm{O}\right) \mathrm{PCl}$ (1) or [ClP $\left.\left(\mathrm{OCH}_{2}\right)_{2}\right]_{2} \mathrm{C}(2)$ with the appropriate propargyl alco- hol in the presence of a base leads to the allenylphosphonates 3-6 and 9-17 (schemes 2 and 3ab) after rearrangement of the initially formed $\mathrm{P}^{\mathrm{III}}$ intermediate; the butadiene 7 was a by-product during the synthesis of $\mathbf{6}$ and species $\mathbf{8}$ is the $\mathrm{P}^{\mathrm{III}}$ intermediate prior to the formation of 9 . The yields reported in these and subsequent schemes are those

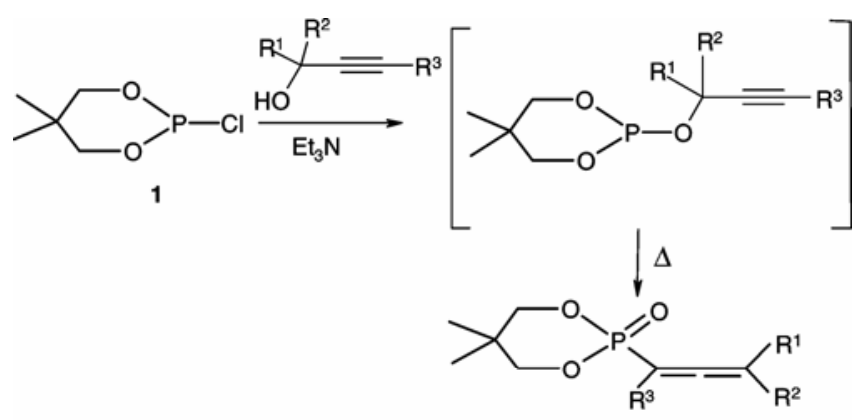

$R^{1}=H, R^{2}=H, R^{3}=\left(C_{2}\right)_{4} C_{3}(3, \delta(P): 11.3 ; 80 \%)$ $R^{1}=H, R^{2}=H, R^{3}=\left(C_{2}\right)_{5} C H_{3}(4, \delta(P): 12.2 ; 78 \%)$ $\mathrm{R}^{1}=\mathrm{H}, \mathrm{R}^{2}=\mathrm{C}(\mathrm{H})=\mathrm{C}(\mathrm{H}) \mathrm{CH}_{3}, \mathrm{R}^{3}=\mathrm{Ph}(5, \delta(\mathrm{P}): 6.7 ; 70 \%)$

\section{Scheme 2.}

(a)
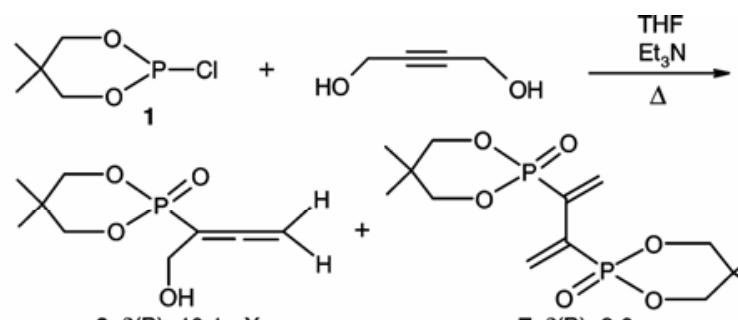

6, $\delta(P): 10.1 ;$ X-ray

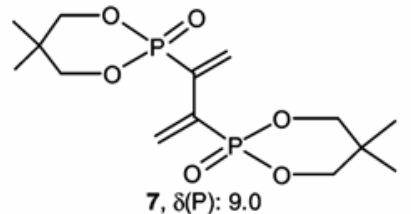

Yield: $80 \%$ ( ${ }^{31} \mathrm{P} \mathrm{NMR}$ ); $17 \%$ (isolated) Yield: $20 \%$ ( ${ }^{31} \mathrm{P}$ NMR); $5 \%$ (isolated) (see text for details)

(b)
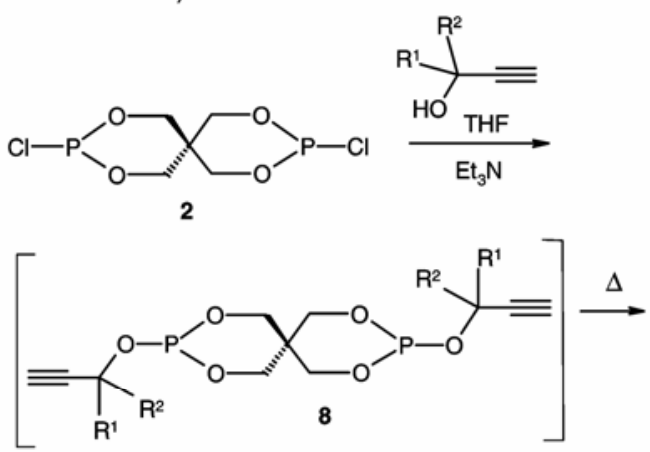

[ $8\left(R^{1}=R^{2}=H\right)$ identified spectroscopically; $\delta(P)$ 125.7 ]

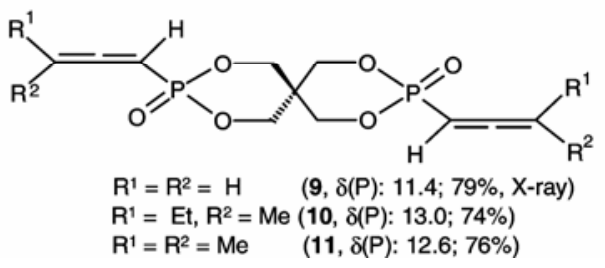

Scheme 3. 


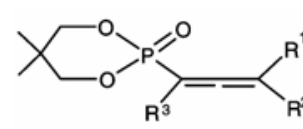

$R^{1}=R^{2}=R^{3}=H(12, \delta(P): 7.4 ; 89 \% ; X$-ray $)$

$R^{1}=R^{3}=H, R^{2}=M e(13, \delta(P): 7.9 ; 86 \%)$

$R^{1}=R^{2}=M e, R^{3}=H(14, \delta(P): 8.5 ; 76 \%)$

$R^{1}=M e, R^{2}=E t, R^{3}=H(15, \delta(P): 9.3 ; 78 \%)$

$R^{1}=H, R^{2}=E t, R^{3}=P h(16, \delta(P): 7.3 ; 84 \%)$

for isolated compounds unless stated otherwise. This type of reaction is known for the synthesis of allenylphosphonates. ${ }^{10}$ A slightly modified procedure was utilized to prepare compounds 9-11 (scheme 3b) and 17 that contain the 1,3,2-dioxaphosphorinane ring with two reactive allene residues.

Although in the reaction using 1 we could not detect the $\mathrm{P}^{\mathrm{III}}$ intermediate alkoxide, we could do so in the synthesis of 9; the species $8\left[R^{1}=R^{2}=H\right]$ was clearly identified by the ${ }^{31} \mathrm{P}$ NMR $[\delta(\mathrm{P})$ 125.7] spectrum as essentially a single component prior to refluxing. Compound 9 was obtained as a crystalline material from methanol; it had low solubility in normal organic solvents like toluene, dichloromethane, etc. Since the $\mathrm{P}^{\mathrm{III}}$ precursor 1 can be prepared by simply stirring phosphorus trichloride with the 1,3-diol for $24 \mathrm{~h}$ followed by distillation in low vacuum, we think that our allenylphosphonates are more convenient to synthesize when compared to several non-phosphorylated allenes. All these allenylphosphonates could be handled in air in general, but over a period of ca $15 \mathrm{~d}, 9-\mathbf{1 1}$ and $\mathbf{1 7}$ turned into liquids. Our attempts to analyse/separate the components of these liquids (column chromatography/ NMR), were not successful.

In the synthesis of 6, the product has to be crystallized carefully because the 1,3-butadiene $\left[\left(\mathrm{OCH}_{2}\right.\right.$ $\left.\left.\mathrm{CMe}_{2} \mathrm{CH}_{2} \mathrm{O}\right)(\mathrm{O}) \mathrm{P}-\mathrm{C}\left(=\mathrm{CH}_{2}\right)\right]_{2}(7)$ was also formed in the same reaction (cf. scheme $3 \mathrm{a}$ ). A chromatographic separation was rendered difficult because of similar polarities of $\mathbf{6}$ and the butadiene by-product 7. We isolated 7 also quantitatively by using $1: 2$ stoichiometry of the propargylic alcohol and $\mathbf{1}$. Since there are a couple of compounds analogous to $7,{ }^{12}$ and since this compound did not react with dimethyl acetylenedicarboxylate (DMAD) or diisopropyl azodicarboxylate (DIAD), we did not explore its chemistry further.

Allenes may be expected to behave as dienophiles in Diels-Alder reaction. Our intention was to incorporate anthracene moiety but when we treated $\mathbf{1 4}$ with anthracene (which is expected to behave as a diene to lead to a [4+2] cycloaddition product) at $185^{\circ} \mathrm{C}$ for $5 \mathrm{~h}$, we obtained only the dimerized allene 18 in a fair yield (scheme 4). Although the reaction mixture showed other products also $\left[{ }^{31} \mathrm{P}\right.$ NMR evidence], we isolated only $\mathbf{1 8}$ and not the expected compound 19. We then tried this reaction in the absence of anthracene and found that $\mathbf{1 8}$ could be obtained in better yields. ${ }^{15}$ The presence of the $\mathrm{PCH}=\mathrm{C}$ moiety in this compound was shown by a doublet at $\delta 5.62\left[{ }^{2} J(\mathrm{P}-\mathrm{H})=16.3 \mathrm{~Hz}\right]$ in the ${ }^{1} \mathrm{H}$ NMR spectrum and a doublet at $\delta 105.2\left({ }^{1} J(\mathrm{P}-\right.$ $\mathrm{C})=186.8 \mathrm{~Hz}$ ) in the ${ }^{13} \mathrm{C}$ NMR spectrum. Only one report on such a reaction is available in the literature, but without an X-ray structural proof. ${ }^{16}$ In our case, we characterized 18 by single crystal Xray diffraction (figure 4). Although the crystal quality was only moderate, the molecular structure was unambiguously determined. The alkenic protons (rather than the phosphonate phosphorus atoms) of the two $\mathrm{C}=\mathrm{CH}\left[\mathrm{P}(\mathrm{O})\left(\mathrm{OCH}_{2} \mathrm{CMe}_{2} \mathrm{CH}_{2} \mathrm{O}\right)\right]$ moieties are in close proximity to each other most likely to avoid steric effects. Since the reaction takes place thermally at elevated temperatures, a diradical mechanism is likely to be involved in the formation of 18; this is similar to the dimerization of many non-phosphorylated allenes. ${ }^{17}$

In our previous paper, we have reported the addition reactions of secondary amines with allenylphosphonates. ${ }^{4}$ In a similar manner, enaminophosphonates 20-21 were prepared by starting with 12-13.

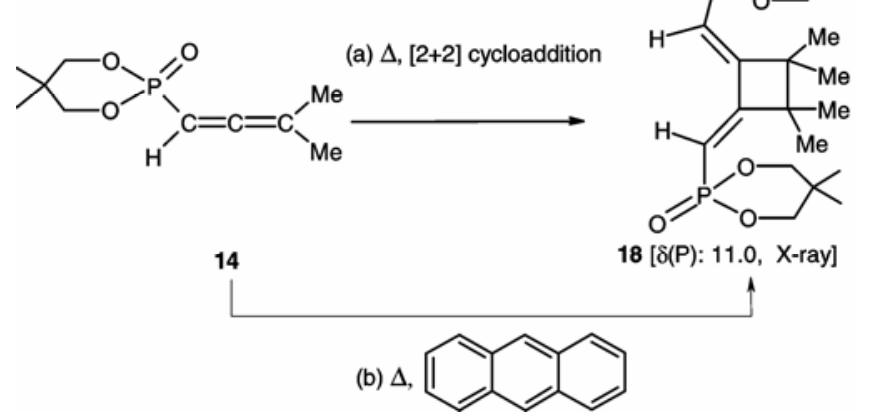

Scheme 4.

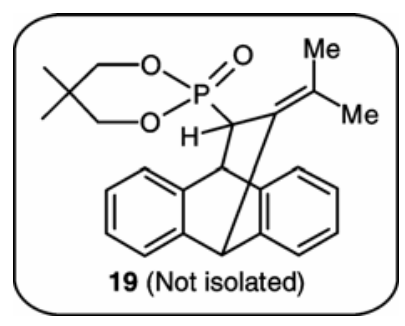


Hydrolysis of 20-21 by $2 \mathrm{~N} \mathrm{HCl}$ afforded the $\beta$ ketophosphonates 22-23 (scheme 5). In reactions using 14-16, we were able to isolate the $\beta$ ketophosphonates 24-26 although the corresponding enaminophosphonates could not be isolated. Addition of $2 \mathrm{~N} \mathrm{HCl}$ to $\mathbf{2 0}$ in an NMR tube experiment showed only 22 along with the starting phosphonate $\left[{ }^{31} \mathbf{P}\right.$ NMR] suggesting that the intermediates [A or B] are very reactive and rapidly lead to $\mathbf{2 2}$ or $\mathbf{2 3}$. Some reports on this type of hydrolysis are known in the literature, ${ }^{18}$ but the present series of compounds are well-defined solids that can be stored indefi-

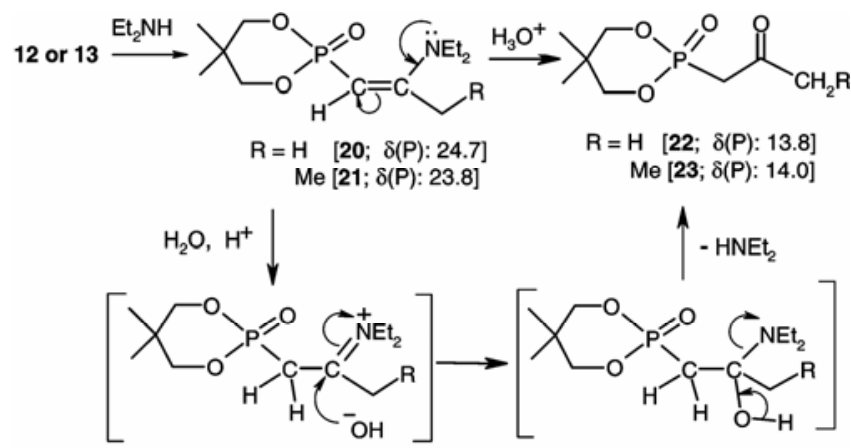

(A)

(B)

Scheme 5.

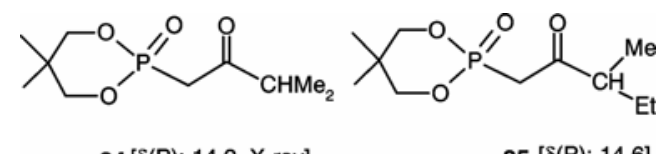

$24[\delta(P): 14.2, X-$ ray $] \quad 25[\delta(P): 14.6]$

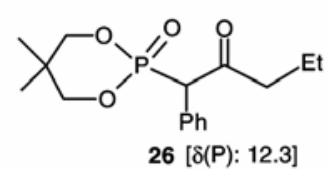

Table 1. ${ }^{31} \mathrm{P}$ NMR chemical shifts for compounds 3-18 and 20-26.

\begin{tabular}{lrcc}
\hline $\begin{array}{l}\text { Compound } \\
\text { no. }\end{array}$ & $\begin{array}{c}\delta(P) \\
(\mathrm{ppm})\end{array}$ & $\begin{array}{c}\text { Compound } \\
\text { no. }\end{array}$ & $\begin{array}{c}\delta(P) \\
(\mathrm{ppm})\end{array}$ \\
\hline $\mathbf{3}$ & $11 \cdot 3$ & $\mathbf{1 5}$ & $9 \cdot 3$ \\
$\mathbf{4}$ & $12 \cdot 2$ & $\mathbf{1 6}$ & $7 \cdot 3$ \\
$\mathbf{5}$ & $6 \cdot 7$ & $\mathbf{1 7}$ & $10 \cdot 6$ \\
$\mathbf{6}$ & $10 \cdot 1$ & $\mathbf{1 8}$ & $11 \cdot 0$ \\
$\mathbf{7}$ & $9 \cdot 0$ & $\mathbf{2 0}$ & $24 \cdot 7$ \\
$\mathbf{8}$ & $125 \cdot 7$ & $\mathbf{2 1}$ & $23 \cdot 8$ \\
$\mathbf{9}$ & $11 \cdot 4$ & $\mathbf{2 2}$ & $13 \cdot 8$ \\
$\mathbf{1 0}$ & $13 \cdot 0$ & $\mathbf{2 3}$ & $14 \cdot 0$ \\
$\mathbf{1 1}$ & $12 \cdot 6$ & $\mathbf{2 4}$ & $14 \cdot 2$ \\
$\mathbf{1 2}$ & $7 \cdot 4$ & $\mathbf{2 5}$ & $14 \cdot 6$ \\
$\mathbf{1 3}$ & $7 \cdot 9$ & $\mathbf{2 6}$ & $12 \cdot 3$ \\
$\mathbf{1 4}$ & $8 \cdot 5$ & & \\
\hline
\end{tabular}

nitely in air. In the IR spectra, they show a band at $\sim 1715 \mathrm{~cm}^{-1}$ corresponding to the carbonyl stretch. In the ${ }^{1} \mathrm{H}$ NMR, a characteristic doublet at $\delta 3.3-3.5$ $\left[{ }^{2} J(\mathrm{P}-\mathrm{H}) \sim 22 \cdot 0 \mathrm{~Hz}\right]$ for the $\mathrm{PCH}_{2}$ protons clearly shows that a rearrangement of the carbon skeleton has taken place. The ${ }^{31} \mathrm{P}$ NMR spectra show a single peak in the range $\delta 12-15$ that are distinctly downfield to those of the parent allenes [ $\delta 7-10]$. For ease of comparison, the ${ }^{31} \mathrm{P}$ NMR data of compounds 318 and 20-26 are given in table 1 . The carbon $\alpha$ to phosphorus appears as a doublet around $\delta 40.0$ in the ${ }^{13} \mathrm{C}$ NMR spectrum; the magnitude of ${ }^{1} J(\mathrm{P}-\mathrm{C})$ $[122.5 \mathrm{~Hz}]$ clearly shows that this carbon has less $s$ character than the corresponding one in 12-16 $\left[{ }^{1} J(\mathrm{P}-\mathrm{C}) 190-200 \mathrm{~Hz}\right]$.

Compounds 22-26 are interesting reagents for Horner-Wadsworth-Emmons (HWE) reaction. ${ }^{19}$ They can also be used as ketonic substrates. ${ }^{20}$ Our initial attempts of the HWE reaction using NaH/THF gave either none or only very low yields of olefinic products. However, when $\mathrm{K}_{2} \mathrm{CO}_{3}$ was used in place of $\mathrm{NaH}$ in refluxing THF, the reaction using 22-23 proceeded smoothly to afford the $\alpha, \beta$ -

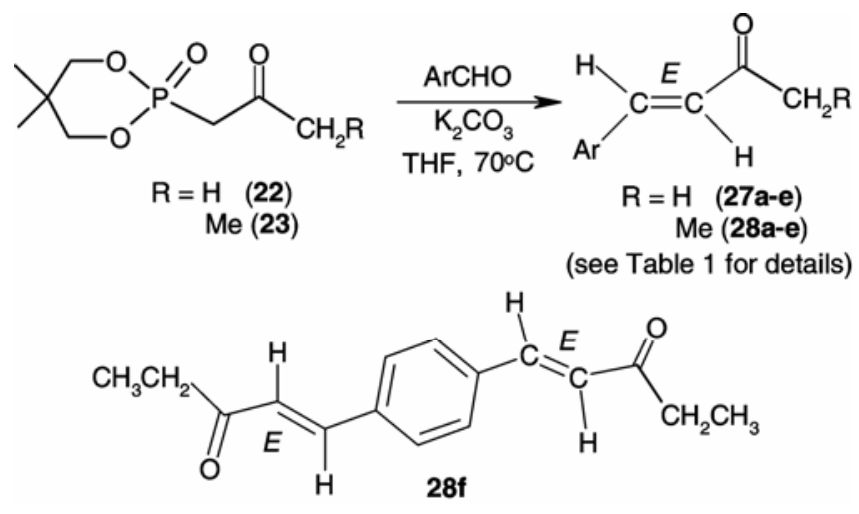

Scheme 6.

Table 2. Details on the HWE reaction of the $\beta$-ketophosphonates 22-23 with aromatic aldehydes.

\begin{tabular}{cllcc}
\hline Entry & $\mathrm{R}$ & \multicolumn{1}{c}{$\mathrm{Ar}$} & Product $(E)$ & $\begin{array}{c}\text { Isolated } \\
\text { yield (\%) }\end{array}$ \\
\hline 1 & $\mathrm{H}$ & $4-\mathrm{Me}-\mathrm{C}_{6} \mathrm{H}_{4}$ & $\mathbf{2 7 a}$ & 79 \\
2 & $\mathrm{H}$ & $3-\mathrm{Me}-\mathrm{C}_{6} \mathrm{H}_{4}$ & $\mathbf{2 7 b}$ & 81 \\
3 & $\mathrm{H}$ & $4-\mathrm{MeO}-\mathrm{C}_{6} \mathrm{H}_{4}$ & $\mathbf{2 7 c}$ & 87 \\
4 & $\mathrm{H}$ & $3,4-\mathrm{Cl}_{2}-\mathrm{C}_{6} \mathrm{H}_{3}$ & $\mathbf{2 7 d}$ & 78 \\
5 & $\mathrm{H}$ & $9-$ anthryl & $\mathbf{2 7 e}$ & 78 \\
6 & $\mathrm{Me}$ & $4-\mathrm{Me}-\mathrm{C}_{6} \mathrm{H}_{4}$ & $\mathbf{2 8 a}$ & 73 \\
7 & $\mathrm{Me}$ & $4-\mathrm{MeO}-\mathrm{C}_{6} \mathrm{H}_{4}$ & $\mathbf{2 8 b}$ & 89 \\
8 & $\mathrm{Me}$ & PhCH= $\mathrm{CH}$ & $\mathbf{2 8 c}$ & 65 \\
9 & $\mathrm{Me}$ & $9-$ anthryl & $\mathbf{2 8 d}$ & 68 \\
10 & $\mathrm{Me}$ & ferrocenyl & $\mathbf{2 8 e}$ & 68 \\
11 & $\mathrm{Me}$ & $4-\mathrm{CHO}-\mathrm{C}_{6} \mathrm{H}_{4}$ & $\mathbf{2 8 f}$ & 79 \\
\hline
\end{tabular}


unsaturated ketones $27 \mathbf{a}-\mathbf{e}$ and $28 \mathbf{a}-\mathbf{f}$ in moderate to high yields (scheme 6 , table 2 ). It is true that aldol condensation is the more straightforward route to $\alpha, \beta$-unsaturated ketones than this, but in specific cases where a particular stereo-/region-chemistry of the products is required, our procedure offers as an alternative.

The $\beta$-ketophosphonates 22-23 also undergo allylation readily with diallyltin dibromide at room tem-

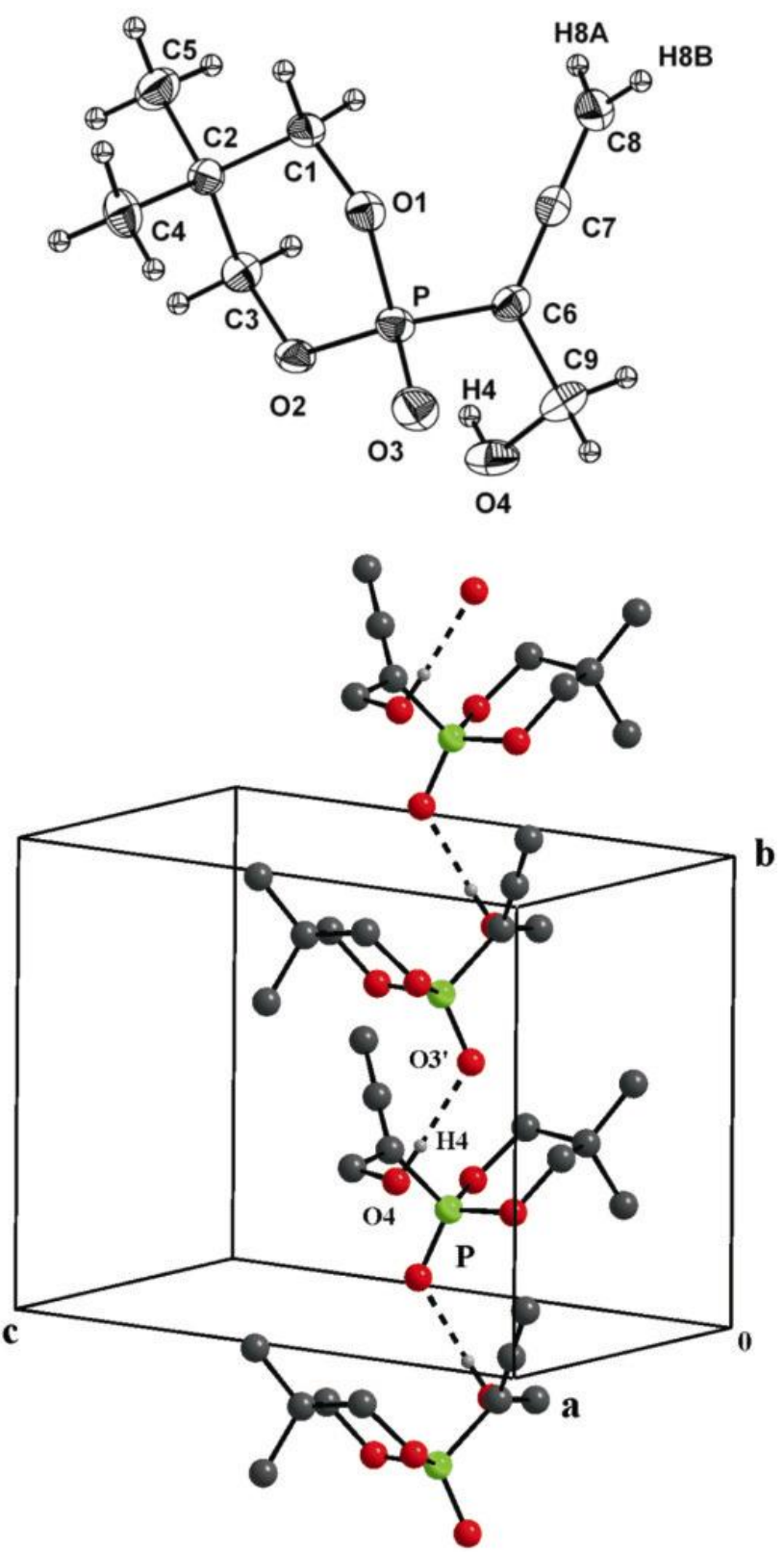

Figure 1. Top: The molecular structure of 6 showing the atom numbering scheme at $30 \%$ probability level of the thermal ellipsoids. Bottom: hydrogen bonding in 6 $\left[\mathrm{O}(4)-\mathrm{H}(4) \ldots \mathrm{O}\left(3^{\prime}\right) 0.82,1.95,2.764(2) \AA, 174.7^{\circ}\right.$. Symmetry code: $1 \cdot 5-x, 0 \cdot 5+y, 0 \cdot 5-\mathrm{z}]$. perature in dichloromethane in the presence of molecular sieves (4 $\AA$ ) to give $\beta$-hydroxyphosphonates $\left(\mathrm{OCH}_{2} \mathrm{CMe}_{2} \mathrm{CH}_{2} \mathrm{O}\right) \mathrm{P}(\mathrm{O}) \mathrm{CH}_{2} \mathrm{C}(\mathrm{OH})\left(\mathrm{CH}_{2} \mathrm{CH}=\mathrm{CH}\right)$ $\mathrm{CH}_{2} \mathrm{R}[\mathrm{R}=\mathrm{H}$ (29), Me (30)]. Although similar indium mediated allylation reactions have been reported before, ${ }^{19}$ our precursors may offer an advantage because they are relatively easier to synthesize.

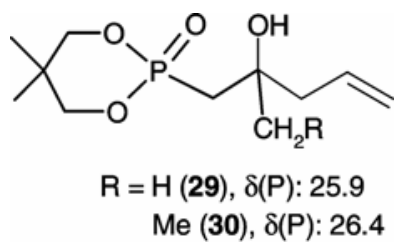

3.1 A brief discussion of the structures of 6, 9, 12, 18 and 24

The structures of 6,9 and $\mathbf{1 2}$ are shown in figures 13 ; only half of the molecule is present in the asym-

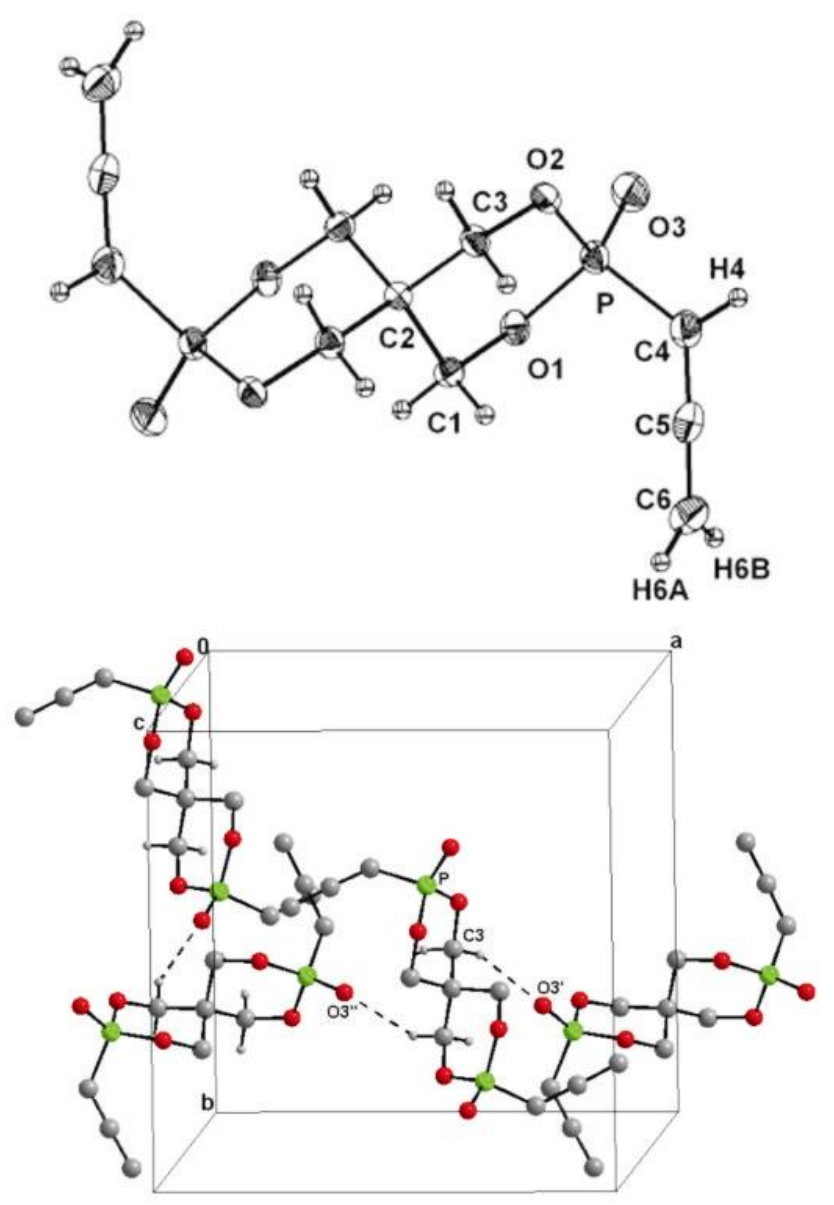

Figure 2. Top: The molecular structure of 9 showing the atom numbering scheme at $30 \%$ probability level of the thermal ellipsoids. Bottom: hydrogen bonding in 9 $\left[\mathrm{C}(3)-\mathrm{H}(3 \mathrm{~A}) \ldots \mathrm{O}\left(3^{\prime}\right) \quad 0.97,2 \cdot 41,3 \cdot 286(4) \AA, \quad 150 \cdot 3^{\circ}\right.$. Symmetry code: (i) $3 / 4+y, 3 / 4-x,-1 / 4+z)]$. 
Table 3. Selected bond parameters for 6,9 and $12(\AA)$.

\begin{tabular}{|c|c|c|c|}
\hline Parameter & 6 & 9 & 12 \\
\hline $\mathrm{P}-\mathrm{O}$ (ring) & $1 \cdot 565(2)$ & $1 \cdot 569(1)$ & $1 \cdot 583(2)$ \\
\hline $\mathrm{P}-\mathrm{O}$ (ring) & $1.573(2)$ & $1 \cdot 568(1)$ & $1 \cdot 588(3)$ \\
\hline $\mathrm{P}=\mathrm{O}$ & $1.456(2)$ & $1 \cdot 466(1)$ & $1.475(3)$ \\
\hline $\mathrm{P}-\mathrm{C}$ & $1.771(2)$ & $1.779(2)$ & $1.777(4)$ \\
\hline $\mathrm{C}(\mathrm{P})=\mathrm{C}$ & $1 \cdot 288(3)$ & $1 \cdot 302(2)$ & $1 \cdot 306(6)$ \\
\hline $\mathrm{C}=\mathrm{CH}_{2}$ & $1 \cdot 280(4)$ & $1 \cdot 290(3)$ & $1 \cdot 307(6)$ \\
\hline $\mathrm{C}=\mathrm{C}=\mathrm{C}$ & $179 \cdot 1(2)$ & $178 \cdot 8(2)$ & $178 \cdot 7(5)$ \\
\hline
\end{tabular}

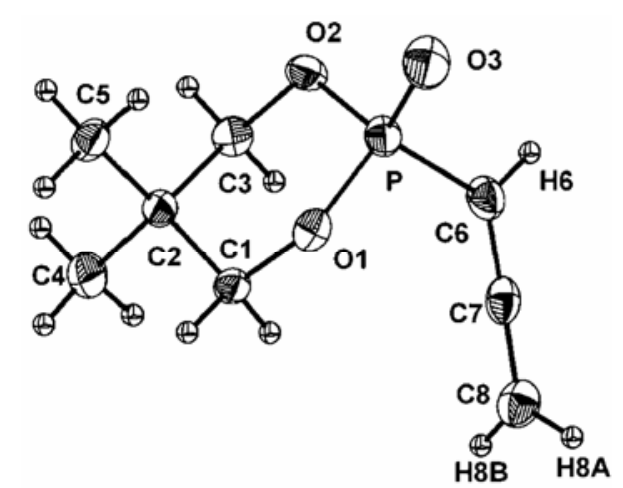

Figure 3. An ORTEP drawing of 12 (see table 3 for bond parameters).

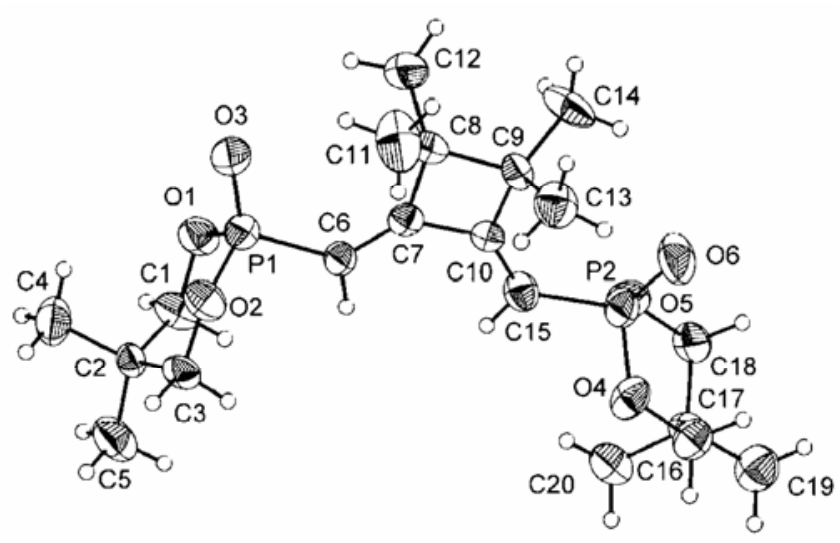

Figure 4. An ORTEP picture of compound 18. Selected bond lengths $(\AA)$ and angles $\left(^{\circ}\right)$ with esd's in parentheses: $\mathrm{P}(1)-\mathrm{C}(6) \quad 1.763(7), \quad \mathrm{P}(2)-\mathrm{C}(15) \quad 1.761(7), \quad \mathrm{C}(6)-\mathrm{C}(7)$ $1.331(8), \mathrm{C}(7)-\mathrm{C}(8) 1.518(9), \mathrm{C}(8)-\mathrm{C}(9) 1.577(9), \mathrm{C}(9)-$ $\mathrm{C}(10) 1.514(8), \mathrm{C}(10)-\mathrm{C}(7) \quad 1.458(9) ; \mathrm{C}(7)-\mathrm{C}(8)-\mathrm{C}(9)$ $88 \cdot 1(5), \quad \mathrm{C}(8)-\mathrm{C}(9)-\mathrm{C}(10) \quad 87 \cdot 0(5), \quad \mathrm{C}(7)-\mathrm{C}(10)-\mathrm{C}(9)$ $92 \cdot 7(5), \mathrm{C}(10)-\mathrm{C}(7)-\mathrm{C}(8) 91 \cdot 3(5)$.

metric unit of 9. Selected bond parameters are given in table 3. The $\mathrm{P}-\mathrm{O}$ (ring), $\mathrm{P}=\mathrm{O}$ and $\mathrm{P}-\mathrm{C}$ distances are in the normal range expected for these compounds. ${ }^{8 \mathrm{a}-\mathrm{b}}$ In contrast to many previously reported

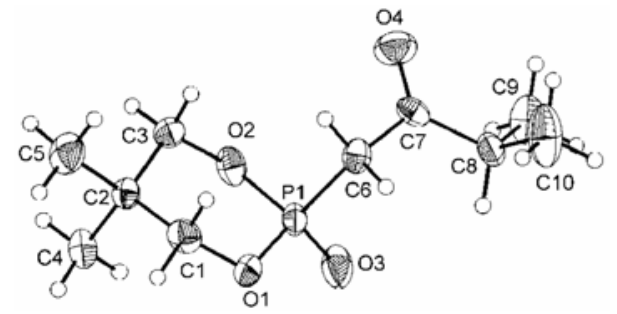

Figure 5. An ORTEP drawing of 24. Only one of the two molecules in asymmetric unit is shown. Selected bond lengths $(\AA)$ with esd's in parentheses: $\mathrm{P}(1)-\mathrm{C}(6)$ $1.802(5), \mathrm{C}(6)-\mathrm{C}(7) 1.509(8), \mathrm{C}(7)-\mathrm{O}(4) 1.193(6), \mathrm{C}(7)-$ $\mathrm{C}(8) 1.507(8)$.

structures, ${ }^{8 \mathrm{~b}-\mathrm{e}}$ the two adjacent $\mathrm{C}=\mathrm{C}$ bonds of the allenyl group are much closer in length, a feature similar to that reported by Angelov et al [1.296(4) and $1.299(4) \AA] .{ }^{8 a}$ The $\mathrm{C}=\mathrm{C}=\mathrm{C}$ unit is essentially linear in all the compounds reported here with a deviation of $\sim 1.3^{\circ}$ from $180^{\circ}$. The dihedral angle between the planes containing (i) $\mathrm{H}(8 \mathrm{~A}), \mathrm{H}(8 \mathrm{~B}), \mathrm{C}(8)$ and $\mathrm{C}(7)$, and (ii) $\mathrm{P}, \mathrm{C}(6), \mathrm{C}(7)$ and $\mathrm{C}(9)$ in compound 6 is $83(1)^{\circ}$ which is significantly different from the expected orthogonality. In 9 and 12, the analogous dihedral angles are, respectively, 87(1) and $89(1)^{\circ}$. The six-membered 1,3,2-dioxaphosphorinane ring has a chair conformation in these structures. Compound 9 has a spirocyclic carbon center; the angles at this center are within $2^{\circ}$ of the ones required for tetrahedral geometry.

As expected, hydrogen-bonding between the phosphoryl oxygen and the -OH group is present in 6. This leads to chain type of structure (figure 1, bottom). In 9, there are weak $\mathrm{C}-\mathrm{H}$... O interactions between the $\mathrm{OCH}_{2}$ protons of the six-membered phosphorinane ring and the phosphoryl oxygen as shown in figure 2 (bottom). Thus each oxygen atom has close contacts with the protons of different methylene groups in the extended structure.

There are two molecules in the asymmetric unit of 18 and only one of these is shown in figure 4. The cyclobutane ring in $\mathbf{1 8}$ can be considered to be planar. The distances $\mathrm{C}(6)-\mathrm{C}(7)$ and $\mathrm{C}(10)-\mathrm{C}(15)$ are clearly in the double bond region but the corresponding $\mathrm{P}-\mathrm{C}$ single bond distances are quite short. In fact, these bonds are shorter than even that seen in the allenes 6,9 and 12. It may be noted that two orientations are possible for the phosphonate moiety with respect to the $\mathrm{C}=\mathrm{C}$, but in the structure, the one that places the two phosphorus atoms with less steric interactions is observed. Compound 24, interestingly, crystallizes in the chiral space group $P 2_{1}$ with two molecules in the asymmetric unit. In this $\beta$ - 
ketophosphonate (cf. figure 5), the phosphorus is connected to an $\mathrm{sp}^{3}$ carbon, and hence the $\mathrm{P}-\mathrm{C}$ bond distance is longer than those in the structures discussed above. Other structural parameters are normal.

\section{Summary}

In summary, we have described the synthesis of several readily accessible and fairly stable solid allenylphosphonates that can be used as potential candidates for exploring allene chemistry; two of these bear additional functionalities like $-\mathrm{OH}$ or $\mathrm{CH}=\mathrm{CHMe}$. Bis-allenylphosphonates of type 9-11 and $\mathbf{1 7}$ may be useful precursors for polymerization. An allene dimer (18) that contains a substituted cyclobutane ring has been structurally characterized. Horner-Wadsworth-Emmons reaction using the $\beta$ ketophosphonates derived from allenylphosphonates serves as an alternative (compared to aldol condensation) source for $\alpha, \beta$-unsaturated ketones.

\section{Acknowledgements}

We thank the Department of Science and Technology (DST, New Delhi) for funding and for setting up a Single Crystal X-ray Diffractometer Facility at the University of Hyderabad and for financial support. Authors also thank University Grants Commission UGC (New Delhi) for equipment under the UPE program. NNBK, MC, NSK, KVS thank Council of Scientific and Industrial Research (CSIR) for fellowships.

\section{References}

1. Selected reviews: (a) Yamamoto $\mathrm{Y}$ and Radhakrishnan U 1999 Chem. Soc. Rev. 28 199; (b) Zimmer R, Dinesh C U, Nandanan E and Khan F A 2000 Chem. Rev. 100 3067; (c) Lu X, Zhang C and Xu Z 2001 Acc. Chem. Res. 34 535; (d) Ma S 2005 Chem. Rev. 105 2829; (e) Hassan H H A M 2007 Current Organic Synthesis 4 413; (f) Brummond K M and DeForrest J E 2006 Synthesis 795

2. Some key references: (a) Alabugin I V and Brel V K 1997 Russ. Chem. Rev. 66 205; (b) Nishimura T, Hirabayashi $\mathrm{S}$ and Hayashi $\mathrm{T} 2006 \mathrm{~J}$. Am. Chem. Soc. 126 2556; (c) Ma S, Guo H and Yu F 2006 J. Org. Chem. 716634

3. (a) Kumara Swamy K C, Kumaraswamy S, Senthil Kumar K, Muthiah C 2005 Tetrahedron Lett. 46 3347; (b) Kommana P, Satish Kumar N, Vittal J J, Jayasree E G, Jemmis E D, Kumara Swamy K C 2004 Org. Lett. 6145
4. (a) Chakravarty M and Kumara Swamy K C 2006 J. Org. Chem. 71 9128; (b) Kumara Swamy K C, Balaraman E and Satish Kumar N 2006 Tetrahedron 6210152

5. Bhuvan Kumar N N and Kumara Swamy K C 2007 Polyhedron 26883

6. Chakravarty M and Kumara Swamy K C 2007 Synthesis 3171

7. Chakravarty M, Bhuvan Kumar N N, Sajna K V and Kumara Swamy K C 2008 Eur. J. Org. Chem. 4500

8. (a) Angelov C M, Mazzuca D A, van den Heever J P, McDonald R, McEwen A J B and Mercer J R 2002 Acta Cryst. $\mathbf{E 5 8}$ 0399; (b) Xu W-M, Hu X-R and Gu J-M 2004 Acta Cryst. E60 02214; (c) Ansorge M, Polborn K and Muller T J J 1999 Eur. J. Inorg. Chem. 225; (d) Muller T J J and Ansorge M 1997 Chem. Ber. 130 1135; (e) Santelli-Rouvier C, Toupet L and Santelli M 1997 J. Org. Chem. 629039

9. Perrin D D, Armarego W L F and Perrin D R 1986 Purification of Laboratory Chemicals (Oxford, UK: Pergamon)

10. (a) Guillemin J C, Savignac P and Denis J M 1991 Inorg. Chem. 30 2170; (b) Iorga B, Eymery F, Carmichael D and Savignac P 2000 Eur. J. Org. Chem. 3103; (c) Satish Kumar N 2004 Studies on the synthesis, reactivity and utility of cyclic phosphorus(III) compounds and organophosphonates $\mathrm{Ph} \mathrm{D}$ thesis, University of Hyderabad, India

11. Fang X-M, Wang Y, Liu Z-G, Xu Y-Q and Ding T 2004 Huaxue Yanjiu 15 43; Chemical Abstr. 141 296087

12. (a) Pollok $\mathrm{T}$ and Schmidbaur $\mathrm{H} 1987$ Tetrahedron Lett. 28 1085; (b) Pudovik A N and Aladzheva I M 1963 Zh. Obshch. Khim. 33 708; Chemical Abstr. 59, 15743

13. Sisido K and Takeda Y 1961 J. Org. Chem. 262301

14. (a) Sheldrick G M SADABS, Siemens Area Detector Absorption Correction, University of Göttingen, Germany, 1996; (b) Sheldrick G M SHELXTL NT Crystal Structure Analysis Package, Bruker AXS, Analytical X-ray System, WI, USA, 1999, version 5.10

15. We sincerely thank a referee for constructive suggestions in this regard.

16. Trifonov L S, Simove S D and Orahovats A S 1987 Tetrahedron Lett. 283391

17. Murakami M and Matsuda T 2004 in Modern allene chemistry (eds) N Krause and A S K Hashmi (Weinheim: Wiley-VCH) Vol 2, Chapter 12, pp 727816

18. Altenbach H and Korff R 1981 Tetrahedron Lett. 22 5175

19. See, for example: (a) Abiko A and Masamune S 1996 Tetrahedron Lett. 37 1077; (b) Muthiah C, Kumar K P, Kumaraswamy S and Kumara Swamy K C 1998 Tetrahedron 54 14315; (b) Chakravarty M, Srinivas B, Muthiah C and Kumara Swamy K C 2003 Synthesis 2368

20. Ranu B C, Samanta S and Hajra A $2001 \mathrm{~J}$. Org. Chem. 667519 